\title{
Smooth measures and capacities associated with nonlocal parabolic operators
}

\author{
TOMASZ KLIMSIAK AND ANDRZEJ ROZKOSZ
}

Abstract. We consider a family $\left\{L_{t}, t \in[0, T]\right\}$ of closed operators generated by a family of regular (non-symmetric) Dirichlet forms $\left\{\left(B^{(t)}, V\right), t \in[0, T]\right\}$ on $L^{2}(E ; m)$. We show that a bounded (signed) measure $\mu$ on $(0, T) \times E$ is smooth, i.e. charges no set of zero parabolic capacity associated with $\frac{\partial}{\partial t}+L_{t}$, if and only if $\mu$ is of the form $\mu=f \cdot m_{1}+g_{1}+\partial_{t} g_{2}$ with $f \in L^{1}((0, T) \times E ; \mathrm{d} t \otimes m), g_{1} \in L^{2}\left(0, T ; V^{\prime}\right)$, $g_{2} \in L^{2}(0, T ; V)$. We apply this decomposition to the study of the structure of additive functionals in the Revuz correspondence with smooth measures. As a by-product, we also give some existence and uniqueness results for solutions of semilinear equations involving the operator $\frac{\partial}{\partial t}+L_{t}$ and a functional from the dual $\mathcal{W}^{\prime}$ of the space $\mathcal{W}=\left\{u \in L^{2}(0, T ; V): \partial_{t} u \in L^{2}\left(0, T ; V^{\prime}\right)\right\}$ on the right-hand side of the equation.

\section{Introduction}

In the study of parabolic problems of the form

$$
-\partial_{t} u-\Delta_{p} u=f(\cdot, u)+\mu \quad \text { in } D,\left.\quad u\right|_{(0, T) \times \partial D}=0, \quad u(T, \cdot)=\varphi,
$$

where $D$ is a bounded open set in $\mathbb{R}^{d}, \Delta_{p}$ is the usual $p$-Laplacian, $p>1$, and $\mu$ is a bounded measure on $D_{0, T}:=(0, T) \times D$ charging no set of zero parabolic $p$-capacity associated with $\frac{\partial}{\partial t}-\Delta_{p}$ (see below) an important role is played by the result on the decomposition of $\mu$ proved by Droniou et al. [6] (see, e.g., [6,24,25]; note that in these papers more general than $\Delta_{p}$ operators of the form $A(u)=\operatorname{div} a(t, x, \nabla u)$ are considered). The decomposition proved in [6] says that each such measure $\mu$ (we call it diffuse) is of the form

$$
\mu=f+\operatorname{div}(G)+\partial_{t} g
$$

for some $f \in L^{1}\left(D_{0, T}\right), G \in\left(L^{p^{\prime}}\left(D_{0, T}\right)\right)^{d}$ and $g \in L^{p}\left(0, T ; W_{0}^{1, p}(D) \cap L^{2}(D)\right)$. Recently, in [16], the converse to this result was proved. The decomposition (1.2) is a counterpart to the decomposition of diffuse measures proved in the stationary case by Boccardo et al. [3]. The decomposition of [3] was extended to the Dirichlet forms setting in [15]. 
There has recently been increasing interest in semilinear evolution problems of the form

$$
-\frac{\partial u}{\partial t}-L_{t} u=f(\cdot, u)+\mu, \quad u(T, \cdot)=\varphi,
$$

involving operators $L_{t}$ associated with a (possibly nonlocal) Dirichlet form and bounded measure that do not charge the sets of zero parabolic capacity associated with $\partial_{t}+L_{t}$ (see [12-14] and the references therein). Motivated by possible applications to problems of the form (1.3), in the present paper we investigate the structure of such measures. We extend the results of [15] to the parabolic setting and at the same time the results of [6] with $p=2$ to more general parabolic operators. As a by-product, we obtain some results on the existence of solutions to equations of the form (1.3) with $\mu \in \mathcal{W}_{0}^{\prime}$ and on the structure of additive functionals associated in the Revuz sense with bounded smooth measures.

Let $E$ be a locally compact separable metric space, $E_{0, T}:=(0, T) \times E$ for some $T>$ 0 , and let $m$ be a Radon measure on $E$ such that $\operatorname{supp}[m]=E$. In the paper we consider smooth measures with respect to parabolic capacities associated with a family $\left\{L_{t}, t \in\right.$ $[0, T]\}$ of closed operators generated by a family $\left\{\left(B^{(t)}, V\right), t \in[0, T]\right\}$ of regular (non-symmetric) Dirichlet forms on $L^{2}(E ; m)$, with common domain $V$, satisfying some mild regularity assumptions. Our general Dirichlet forms setting allows us to treat both local and nonlocal operators. The results of the paper are new even for local operators. However, in our opinion, the most interesting fact is that we are able to describe the structure of smooth measures (and related additive functionals) for capacities associated with quite large class of parabolic nonlocal operators.

The model example of the family of local operators satisfying our assumptions is the family of divergence form operators

$$
L_{t}=\frac{1}{2} \sum_{i, j=1}^{d} \frac{\partial}{\partial x_{i}}\left(a_{i j}(t, x) \frac{\partial}{\partial x_{j}}\right), \quad t \in[0, T], \quad x \in D,
$$

on $L^{2}(D ; \mathrm{d} x)$ with zero Dirichlet boundary conditions. In (1.4), $D$ is a bounded open subset of $\mathbb{R}^{d}$ and $\left\{a_{i j}(t, x)\right\}_{i, j=1, \ldots, d}$ is a symmetric uniformly elliptic matrix with bounded measurable elements. In this case the family $\left\{\left(B^{(t)}, V\right), t \in[0, T]\right\}$ is of the form

$$
B^{(t)}(u, v)=\sum_{i, j=1}^{d} \int_{D} a_{i j}(t, x) \frac{\partial u}{\partial x_{i}}(x) \frac{\partial v}{\partial x_{j}}(x) \mathrm{d} x, \quad u, v \in V:=H_{0}^{1}(D) .
$$

A model example of the family of nonlocal operators satisfying our assumptions is the family consisting of single fractional Laplace operator

$$
L_{t}=-(-\Delta)^{\alpha / 2}
$$


on $L^{2}(D ; \mathrm{d} x)$ with zero exterior condition. Here $\alpha \in(0,2)$ and $D$ is an open subset of $\mathbb{R}^{d}$. In this case $\left(B^{(t)}, V\right)=(B, V), t \in[0, T]$, where

$B(u, v)=\int_{\mathbb{R}^{d}} \hat{u}(x) \overline{\hat{v}}(x)|x|^{\alpha} \mathrm{d} x, \quad u, v \in V:=\left\{w \in L^{2}(D ; \mathrm{d} x): \int_{\mathbb{R}^{d}}|\hat{u}|^{2}|x|^{\alpha} \mathrm{d} x<\infty\right\}$

and $\hat{u}$ (resp. $\hat{v}$ ) is the Fourier transform of $u$ (resp. $v$ ) (see [8, Example 1.4.1, Theorem 4.4.3]).

In case of problem (1.1), the natural capacity is the $p$-parabolic capacity cap $p$ defined for open set $U \subset D_{0, T}$ by

$$
\operatorname{cap}_{p}(U)=\inf \left\{\left\|\partial_{t} u\right\|_{L^{p^{\prime}}\left(0, T ; V_{p}^{\prime}\right)}+\|u\|_{L^{p}\left(0, T ; V_{p}\right)}: u \geq \mathbf{1}_{U} \mathrm{~d} t \otimes \mathrm{d} x \text {-a.e. }\right\}
$$

where $V_{p}=W_{0}^{1, p} \cap L^{2}(D)$ and $V_{p}^{\prime}$ is the dual of $V_{p}$ (see $[6,28]$ ). To study evolution problems with the operator $\partial_{t}+L_{t}$, Pierre [28] introduced the capacity $c_{2}$ defined for open $U \subset E_{0, T}$ by

$$
c_{2}(U)=\inf \left\{\left\|\partial_{t} u\right\|_{L^{2}\left(0, T ; V^{\prime}\right)}+\left(\int_{0}^{T} B^{(t)}(u(t), u(t)) \mathrm{d} t\right)^{1 / 2}: u \geq \mathbf{1}_{U} m_{1} \text {-a.e. }\right\},
$$

where $m_{1}=\mathrm{d} t \otimes m$. In the potential theory, Borel measures on $E_{0, T}$ which do not charge sets of zero capacity $c_{2}$ and satisfy some quasi-finiteness condition are called smooth measures. In particular, each bounded Borel measure "absolutely continuous" with respect to $c_{2}$ is smooth. Of course, in case of operators of the form (1.4) the classes of diffuse measures and bounded smooth measures coincide.

In our main theorem we extend (1.2) to the Dirichlet form setting. Let $\mathcal{M}_{0, b}\left(E_{0, T}\right)$ denote the set of all bounded smooth measures on $E_{0, T}$. We show that each $\mu \in$ $\mathcal{M}_{0, b}\left(E_{0, T}\right)$ admits decomposition of the form

$$
\mu=f \cdot m_{1}+g_{1}+\partial_{t} g_{2}
$$

with $f \in L^{1}\left(E_{0, T} ; m_{1}\right), g_{1} \in \mathcal{V}^{\prime}=L^{2}\left(0, T ; V^{\prime}\right), g_{2} \in \mathcal{V}=L^{2}(0, T ; V)$, i.e. for every bounded quasi-continuous $\eta \in \mathcal{W}_{0}=\left\{u \in \mathcal{V}: \partial_{t} u \in \mathcal{V}^{\prime}, u(0)=0\right\}$, we have

$$
\int_{E_{0, T}} \eta \mathrm{d} \mu=\int_{E_{0, T}} f \eta \mathrm{d} t \mathrm{~d} m+\left\langle g_{1}, \eta\right\rangle-\left\langle\partial_{t} \eta, g_{2}\right\rangle,
$$

where $\langle\cdot, \cdot\rangle$ denotes the duality between $\mathcal{V}^{\prime}$ and $\mathcal{V}$. We also show the converse of this theorem. Namely, each $\mu \in \mathcal{M}_{b}\left(E_{0, T}\right)$ having decomposition (1.7) is smooth. Note that the converse is an extension of the result proved by Fukushima [7] to timedependent Dirichlet forms. The proof of the fact that $\mu \in \mathcal{M}_{0, b}\left(E_{0, T}\right)$ can be written in the form (1.7) is purely analytic. Essential to the proof of the converse part are probabilistic methods.

In applications to (1.1), the analysis of additional properties of the term $g$ appearing in the decomposition (1.2) proved to be important. For instance, crucial to the definition and the existence result of a solution $u$ of (1.1) is the fact that $g$ regularizes $u$ with 
respect to time in the sense that $u-g \in W \subset C\left([0, T], L^{p}(D)\right)$, where $W=\{u \in$ $L^{p}\left(0, T ; V_{p}\right): \partial_{t} u \in L^{p^{\prime}}\left(0, T ; V_{p}^{\prime}\right)$. This property together with some other useful properties of $g$ has been proved in [6]. In the present paper, applying the potential theory tools, we show that $g_{2}$ from (1.7) enjoys similar properties. We also show some new results on the regularity of $g_{2}$. We show the following useful properties.

- $g_{2}$ has an $m_{1}$-version $\tilde{g}_{2}$ which is quasi-càdlàg (i.e quasi-right-continuous with left limits; this notion generalizes the notion of quasi-continuity; see Sect. 9) and $g_{2}$ is a difference of $c_{2}$-quasi-l.s.c. functions.

- $g_{2}$ has an $m_{1}$-version $\tilde{g}_{2}$ which is $\mathrm{c}_{2}$-quasi-bounded, i.e. there exists an increasing sequence $\left\{F_{n}\right\}$ of closed subsets of $E_{0, T}$ such that $c_{2}\left(E_{0, T} \backslash F_{n}\right) \rightarrow 0$ as $n \rightarrow \infty$ and $\left\|\tilde{g}_{2} \mathbf{1}_{F_{n}}\right\|_{\infty}<\infty, n \geq 1$.

- The function $[0, T] \ni t \mapsto g_{2}(t) \in L^{1}(E ; \rho \cdot m)$ is càdlàg (right-continuous with left limits) for every positive Borel function $\rho$ on $E$ such that $\int_{E} \rho \mathrm{d} m<\infty$, and $g_{2}(T-):=\lim _{t \rightarrow T^{-}} g_{2}(t)=0$.

- The measure $\mu_{t}$ defined by $\mu_{t}(B)=\mu(\{t\} \times B)$ for Borel sets $B \subset E$ is absolutely continuous with respect to $m$ and $\mu_{t}=\left(g_{2}(t)-g_{2}(t-)\right) \cdot m$.

- If $\mu$ admits decomposition $\mu=f^{\prime} \cdot m_{1}+g_{1}^{\prime}+\partial_{t} g_{2}^{\prime}$ with some $f^{\prime} \in L^{1}\left(E_{0, T} ; m_{1}\right)$, $g_{1}^{\prime} \in \mathcal{V}^{\prime}, g_{2}^{\prime} \in \mathcal{V}$, then the function $[0, T] \ni t \mapsto\left(g_{2}-g_{2}^{\prime}\right)(t) \in L^{1}(E ; \rho \cdot m)$ belongs to $C\left([0, T], L^{1}(E ; \rho \cdot m)\right)$ for every positive Borel function $\rho$ on $E$ such that $\int_{E} \rho \mathrm{d} m<\infty$.

It is worth pointing out here that the proofs of the above results in the general setting requires us to use quite different methods then those used in [6] for the Leray-Lionstype operators, which are strongly based on the regularization of the measure $\mu$ by a convolution operator.

In the proof of our main decomposition theorem, we apply some deep results from the potential theory for evolution operators proved by Pierre [26-28], as well as from the probabilistic potential theory for time dependent or generalized Dirichlet forms developed in the papers by Oshima [20-23], Stannat [30,31] and Trutnau [32,33]. In these papers the definitions of the capacity (and hence some quasi-notions) are different. In Sect. 3, which is technical but important for us, we show that all these capacities are in fact equivalent on $E_{0, T}$. This allows us to apply freely the results from the papers mentioned above.

One of the most important ingredient of the proof that each $\mu \in \mathcal{M}_{b}\left(E_{0, T}\right)$ having decomposition (1.7) is smooth is an existence result for the Cauchy problem (1.3) with $\mu \in \mathcal{W}_{0}^{\prime}$ and $f$ not depending on $u$. If $\mu \in L^{2}\left(0, T ; V^{\prime}\right)$, then the existence of a solution to (1.3) follows from the classical theory of variational inequalities (see [17]). However, if $\mu \in \mathcal{W}_{0}^{\prime}$, then the situation is more difficult. To prove the existence of a solution, a decomposition similar to (1.7), but for functionals from $\mathcal{W}_{0}^{\prime}$ is needed. In case of (1.1), such a decomposition and an existence result were proved in [6]. In Sect. 5, we prove a similar decomposition result for $\mu \in \mathcal{W}_{0}^{\prime}$, and then, in Sect. 6, we deal with the existence of a solution of (1.3) with $\mu \in \mathcal{W}_{0}^{\prime}$. Though for our applications we only need the existence result for liner equations, in the paper we show that there 
exist a solution for semilinear problem (1.3) under the assumption that $\varphi \in L^{2}(E ; m)$ and $u \mapsto f(\cdot, u)$ is continuous, nonincreasing and satisfies the linear growth condition. We think that this result may be of independent interest. Finally, note here that in the proof that $\mu$ given by (1.7) is smooth we use a very recent result from the paper by Beznea and Cîmpean [1] on the characterization of quasimartingale functions.

It is well known that there is a one to one correspondence, called Revuz correspondence, between positive smooth, with respect to the Dirichlet form $(B, V)$, measures on $E$ and positive continuous additive functionals of the Hunt processes associated with $(B, V)$ (see $[8,19])$. In the parabolic case the situation is more subtle. Let $\mathbb{X}$ denote a Hunt process associated with a generalized Dirichlet form $\mathcal{E}$ in the resolvent sense (see Sect. 2) which is generated by the operator $\frac{\partial}{\partial t}+L_{t}$. In the paper we first show that with each smooth measure $\mu$ on $E_{0, T}$ with respect the form $\mathcal{E}$ one can associate uniquely a positive additive functional $A^{\mu}$ of $\mathbb{X}$ which is natural, i.e. has no common discontinuities with the Hunt process associated with $\mathcal{E}$. This is a counterpart to the known result concerning smooth measures on $\mathbb{R} \times E$ (see [21,23]). Then we analyse more carefully the nature of jumps of $A^{\mu}$ in the case where $\mu \in \mathcal{M}_{0, b}\left(E_{0, T}\right)$. Roughly speaking, our main result says that for $\mu \in \mathcal{M}_{0, b}\left(E_{0, T}\right)$ the jumps of $A^{\mu}$ are related to $g_{2}$ from decomposition (1.7). We show that $g_{2}$ has always a quasi-càdlàg modification $\tilde{g}_{2}$, i.e. an $m_{1}$-version $\tilde{g}_{2}$ such that the process $t \mapsto \tilde{g}_{2}\left(X_{t}\right)$ is right-continuous with left limits, and for every predictable stopping time $\tau$,

$$
\Delta A_{\tau}^{\mu}:=A_{t}^{\mu}-A_{t-}^{\mu}=\Delta \tilde{g}_{2}(X)_{\tau} .
$$

In other words, $A^{\mu}$ has jumps that coincide with the jumps of the process $\tilde{g}_{2}(X)$ in predictable stopping times. This implies that $\tilde{g}_{2}$ is quasi-continuous if and only if $A^{\mu}$ is continuous.

\section{Preliminaries}

In this paper, $E$ is a locally compact separable metric space and $m$ is an everywhere dense Radon measure on $E$, i.e. $m$ is a positive Borel measure on $E$, which is finite on compact sets and strictly positive on nonempty open sets.

We set $E^{1}=\mathbb{R} \times E, m_{1}=\mathrm{d} t \otimes m$, and for $T>0$, we set $E_{0, T}=(0, T) \times E$. We denote by $\mathcal{B}\left(E^{1}\right)$ (resp. $\mathcal{B}\left(E_{0, T}\right)$ ) the set of all Borel measurable subsets of $E^{1}$ (resp. $E_{0, T}$ ). With the customary abuse of notation, the same symbols are used to denote the sets of real Borel measurable functions on $E^{1}$ (resp. $E_{0, T}$ ). $\mathcal{B}_{b}\left(E^{1}\right)$ is the set of all real bounded Borel measurable functions on $E^{1}$ and $\mathcal{B}_{b}^{+}\left(E^{1}\right)$ is the subset of $\mathcal{B}_{b}\left(E^{1}\right)$ consisting of positive functions. The sets $\mathcal{B}_{b}\left(E_{0, T}\right), \mathcal{B}_{b}^{+}\left(E_{0, T}\right)$ are defined analogously.

\subsection{Time-dependent Dirichlet forms}

Let $H=L^{2}(E ; m)$ and $(\cdot, \cdot)_{H}$ denote the usual inner product in $H$. In this paper, we assume that we are given a family $\left\{\left(B^{(t)}, V\right), t \in[0, T]\right\}$ of regular (non-symmetric) 
Dirichlet forms on $H$ with common domain $V \subset H$ (see [19, Chapter I] for the definitions) satisfying the following conditions.

(a) There is $K \geq 0$ such that $\left|B_{1}^{(t)}(\varphi, \psi)\right| \leq K\left(B_{1}^{(t)}(\varphi, \varphi)\right)^{1 / 2}\left(B_{1}^{(t)}(\psi, \psi)\right)^{1 / 2}$ for all $\varphi, \psi \in V, t \in[0, T]$, where as usual, we set $B_{\lambda}^{(t)}(\varphi, \psi)=B^{(t)}(\varphi, \psi)+$ $\lambda(\varphi, \psi)_{H}$ for $\lambda \geq 0$.

(b) $[0, T] \ni t \mapsto B^{(t)}(\varphi, \psi)$ is measurable for all $\varphi, \psi \in V$.

(c) There is $c \geq 1$ such that $c^{-1} B^{(0)}(\varphi, \varphi) \leq B^{(t)}(\varphi, \varphi) \leq c B^{(0)}(\varphi, \varphi)$ for all $t \in[0, T]$ and $\varphi \in V$.

To shorten notation, we continue to write $B$ for $B^{(0)}$. By putting $B^{(t)}=B$ for $t \notin$ $[0, T]$, we may and will assume that $B^{(t)}$ is defined and satisfies (c) for all $t \in \mathbb{R}$. We denote by $\tilde{B}^{(t)}$ the symmetric part of $B^{(t)}$, i.e. $\tilde{B}^{(t)}(\varphi, \psi)=\frac{1}{2}\left(B^{(t)}(\varphi, \psi)+\right.$ $\left.B^{(t)}(\psi, \varphi)\right)$.

Since $V$ is a dense subspace of $H$ and $(B, V)$ is closed, $V$ is a real Hilbert space with respect to $\tilde{B}_{1}(\cdot, \cdot)$, which is densely and continuously embedded in $H$. We equip $V$ with the norm $\|\cdot\|_{V}$ defined by $\|\varphi\|_{V}^{2}=B_{1}(\varphi, \varphi), \varphi \in V$. We denote by $V^{\prime}$ the dual space of $V$, and by $\|\cdot\|_{V^{\prime}}$ the corresponding norm. For $T>0$, we set

$$
\mathcal{H}=L^{2}(0, T ; H), \quad \mathcal{V}=L^{2}(0, T ; V), \quad \mathcal{V}^{\prime}=L^{2}\left(0, T ; V^{\prime}\right)
$$

and

$$
\|u\|_{\mathcal{V}}^{2}=\int_{0}^{T}\|u(t)\|_{V}^{2} \mathrm{~d} t, \quad\|u\|_{\mathcal{V}^{\prime}}^{2}=\int_{0}^{T}\|u(t)\|_{V^{\prime}}^{2} \mathrm{~d} t .
$$

We shall identify $H$ and its dual $H^{\prime}$. Then $V \subset H \simeq H^{\prime} \subset V^{\prime}$ continuously and densely, and hence $\mathcal{V} \subset \mathcal{H} \simeq \mathcal{H}^{\prime} \subset \mathcal{V}^{\prime}$ continuously and densely.

For given $u \in \mathcal{V}$ let $\partial_{t} u$ denote the derivative in the distribution sense of the function $t \mapsto u(t) \in V$, and let

$$
\mathcal{W}=\left\{u \in \mathcal{V}: \partial_{t} u \in \mathcal{V}^{\prime}\right\}, \quad\|u\|_{\mathcal{W}}=\|u\|_{\mathcal{V}}+\left\|\partial_{t} u\right\|_{\mathcal{V}^{\prime}}
$$

It is well known that there is a continuous embedding of $\mathcal{W}$ into $C([0, T] ; H)$, i.e. for every $u \in \mathcal{W}$ one can find $\bar{u} \in C([0, T] ; H)$ such that $u(t)=\bar{u}(t)$ for a.e. $t \in[0, T]$ (with respect to the Lebesgue measure) and

$$
\|u\|_{C([0, T] ; H)} \leq C\|u\|_{\mathcal{W}}
$$

for some $C>0$. In what follows, we adopt the convention that any element of $\mathcal{W}$ is already in $C([0, T] ; H)$. With this convention we may define the spaces

$$
\mathcal{W}_{0}=\{u \in \mathcal{W}: u(0)=0\}, \quad \mathcal{W}_{T}=\{u \in \mathcal{W}: u(T)=0\} .
$$

Note that $\mathcal{W}_{0}, \mathcal{W}_{T}$ are reflexive spaces as closed linear subspaces of the reflexive space $\mathcal{W}$.

The linear operator $\partial_{t}$ on $\mathcal{H}$ with domain $\mathcal{W}_{T}$ will be denoted by $\Lambda$. Its adjoint, i.e. the operator $-\partial_{t}$ with domain $\mathcal{W}_{0}$, will be denoted by $\hat{\Lambda}$. 
We denote by $\mathcal{E}$ the generalized Dirichlet form associated with $\Lambda$ and the family $\left\{\left(B^{(t)}, V\right), t \in[0, T]\right\}$, that is

$$
\mathcal{E}(u, v)= \begin{cases}\left\langle-\partial_{t} u, v\right\rangle+\mathcal{B}(u, v), & u \in \mathcal{W}_{T}, v \in \mathcal{V}, \\ \left\langle\partial_{t} v, u\right\rangle+\mathcal{B}(u, v), & u \in \mathcal{V}, v \in \mathcal{W}_{0}\end{cases}
$$

where $\langle\cdot, \cdot\rangle$ is the duality pairing between $\mathcal{V}^{\prime}$ and $\mathcal{V}$, and

$$
\mathcal{B}(u, v)=\int_{0}^{T} B^{(t)}(u(t), v(t)) \mathrm{d} t .
$$

Let $D(\mathcal{E})=\left(\mathcal{W}_{T} \times \mathcal{V}\right) \cup\left(\mathcal{V} \times \mathcal{W}_{0}\right)$. For every every $\alpha \geq 0$ we set

$$
\mathcal{E}_{\alpha}(u, v):=\mathcal{E}(u, v)+\alpha(u, v)_{\mathcal{H}}, \quad(u, v) \in D(\mathcal{E}) .
$$

The generalized form associated with $\hat{\Lambda}$ and the family $\left\{\left(\hat{B}^{(t)}, V\right), t \in[0, T]\right\}$, where $\hat{B}^{(t)}(u, v)=B^{(t)}(v, u), u, v \in V, t \in[0, T]$, will be denoted by $\hat{\mathcal{E}}$.

In the paper, we denote by $\left(G_{\alpha}\right)_{\alpha>0}$ (resp. $\left.\hat{G}_{\alpha}\right)_{\alpha>0}$ the resolvent (resp. coresolvent) associated with the form $\mathcal{E}$, i.e. $\left(G_{\alpha}\right)_{\alpha>0},\left(\hat{G}_{\alpha}\right)_{\alpha>0}$ are strongly continuous resolvents of contractions on $\mathcal{H}$ such that $G_{\alpha}(\mathcal{H}) \subset \mathcal{W}_{T}, \hat{G}_{\alpha}(\mathcal{H}) \subset \mathcal{W}_{0}$ and

$$
\mathcal{E}_{\alpha}\left(G_{\alpha} f, g\right)=(f, g)_{\mathcal{H}}=\mathcal{E}_{\alpha}\left(g, \hat{G}_{\alpha} f\right), \quad f \in \mathcal{H}, g \in \mathcal{V}
$$

(for a construction of the resolvents see, e.g., [31, Chapter I]).

Let $\mathcal{W}^{1}=\left\{u \in L^{2}(\mathbb{R} ; V): \partial_{t} u \in L^{2}\left(\mathbb{R} ; V^{\prime}\right)\right\}$. We denote by $\mathcal{E}^{1}$ the time-dependent Dirichlet form associated with $\left\{\left(B^{(t)}, V\right), t \in \mathbb{R}\right\}$, that is

$$
\mathcal{E}^{1}(u, v)=\left\{\begin{array}{l}
\left\langle-\partial_{t} u, v\right\rangle+\mathcal{B}^{1}(u, v), \quad u \in \mathcal{W}^{1}, v \in L^{2}(\mathbb{R} ; V), \\
\left\langle\partial_{t} v, u\right\rangle+\mathcal{B}^{1}(u, v), \quad u \in L^{2}(\mathbb{R} ; V), v \in \mathcal{W}^{1},
\end{array}\right.
$$

where now $\langle\cdot, \cdot\rangle$ stands for the duality pairing between $L^{2}\left(\mathbb{R} ; V^{\prime}\right)$ and $L^{2}(\mathbb{R} ; V)$, and

$$
\mathcal{B}^{1}(u, v)=\int_{\mathbb{R}} B^{(t)}(u(t), v(t)) \mathrm{d} t .
$$

Analogously to the form $\mathcal{E}$ we define $\mathcal{E}_{\alpha}^{1}$ for every $\alpha \geq 0$. The resolvent (resp. coresolvent) associated with the form $\mathcal{E}^{1}$ (see [31, Chapter I]) will be denoted by $\left(G_{\alpha}^{1}\right)_{\alpha>0}$ (resp. $\left.\left(\hat{G}_{\alpha}^{1}\right)_{\alpha>0}\right)$.

Note that $\mathcal{E}^{1}$ can be identified with some generalized Dirichlet forms in the sense considered in [31-33] (see [31, Example I.4.9(iii)]).

Let $\psi \in L^{1}\left(E_{0, T} ; m_{1}\right) \cap \mathcal{B}\left(E_{0, T}\right)$ be a function such that $0<\psi \leq 1$. We define the capacity $\operatorname{Cap}_{\psi}$ associated with $\mathcal{E}$ as in [31, Sect. III.2] (see also [33]), that is for an open set $U \subset E_{0, T}$ we set

$$
\operatorname{Cap}_{\psi}(U)=\left(\left(G_{1} \psi\right)_{U}, \psi\right)_{\mathcal{H}},
$$

where $\left(G_{1} \psi\right)_{U}$ is the 1-reduced function of $G_{1} \psi$, and then for arbitrary $A \subset E_{0, T}$ we set $\operatorname{Cap}_{\psi}(A)=\inf \left\{\operatorname{Cap}_{\psi}(U), U \supset A, U\right.$ open\}. By [31, Lemma III.2.9] and (2.4), 
for any open $U \subset E_{0, T},\left(\left(G_{1} \psi\right)_{U}, \psi\right)_{\mathcal{H}}=\mathcal{E}_{1}\left(\left(G_{1} \psi\right)_{U}, \hat{G}_{1} \psi\right)=\mathcal{E}_{1}\left(G_{1} \psi,\left(\hat{G}_{1} \psi\right)_{U}\right)$ $=\left(\psi,\left(\hat{G}_{1} \psi\right)_{U}\right)_{\mathcal{H}}$, so $\operatorname{Cap}_{\psi}$ associated with $\mathcal{E}$ coincides with the capacity defined as $\operatorname{Cap}_{\psi}$ but for the dual form $\hat{\mathcal{E}}$. In particular, $\operatorname{Cap}_{\psi}$ coincides with the capacity considered in [32].

By [31, Proposition III.2.8], $\mathrm{Cap}_{\psi}$ is a Choquet capacity. Also note that by [31, Proposition III.2.10], the family of sets $A \subset E_{0, T}$ such that $\operatorname{Cap}_{\psi}(A)=0$ is the same for all $\psi$ as above.

We will denote by $\mathrm{Cap}^{1}$ the capacity associated with $\mathcal{E}^{1}$ and defined in [22] (the definition is also given in [21] and [23, Sect. 6.2]). By [22, Lemma 4.2] (or [23, Lemma 6.2.8]) and [8, Theorem A.1.2], Cap $^{1}$ is a Choquet capacity.

Some relations between $\mathrm{Cap}_{\psi}$ and $\mathrm{Cap}^{1}$, as well as relations between $\mathrm{Cap}_{\psi}$ and some other notions of parabolic capacity considered in the literature will be studied in Sect. 3.

We say that a set $A \subset E_{0, T}$ (resp. $\left.A \subset E^{1}\right)$ is $\mathcal{E}$-exceptional (resp. $\mathcal{E}^{1}$-exceptional) if $\operatorname{Cap}_{\psi}(A)=0\left(\operatorname{resp} . \operatorname{Cap}^{1}(A)=0\right)$, and we say that a property holds $\mathcal{E}$-quasieverywhere (resp. $\mathcal{E}^{1}$-quasi-everywhere) if the set of those $x \in E_{0, T}$ (resp. $x \in E^{1}$ ) for which it does not hold is $\mathcal{E}$-exceptional (resp. $\mathcal{E}^{1}$-exceptional).

Recall that an increasing sequence $\left\{F_{n}\right\}$ of closed subsets of $E_{0, T}$ (resp. $E^{1}$ ) is called an $\mathcal{E}$-nest (resp. $\mathcal{E}^{1}$-nest) if $\operatorname{Cap}_{\psi}\left(F_{n}^{c}\right) \rightarrow 0\left(\operatorname{resp} . \operatorname{Cap}^{1}\left(F_{n}^{c}\right) \rightarrow 0\right)$ as $n \rightarrow \infty$. An increasing sequence $\left\{F_{n}\right\}$ of closed subsets of $E^{1}$ is called a generalized $\mathcal{E}^{1}$-nest if $\operatorname{Cap}^{1}\left(K \backslash F_{n}\right) \rightarrow 0$ for every compact $K \subset E_{0, T}$.

A function $u: E_{0, T} \rightarrow \mathbb{R}$ (resp. $u: E^{1} \rightarrow \mathbb{R}$ ) is called $\mathcal{E}$-quasi-continuous (resp. $\mathcal{E}^{1}$-quasi-continuous) if there exists an $\mathcal{E}$-nest (resp. $\mathcal{E}$-nest) $\left\{F_{n}\right\}$ such that $\left.u\right|_{F_{n}}$ is continuous for every $n \in \mathbb{N}$.

It is known (see [31, Proposition IV.1.8]) that each $u \in \mathcal{W}_{0}$ has an $\mathcal{E}$-quasicontinuous $m_{1}$-version. Similarly, each $u \in \mathcal{W}_{T}$ has an $\mathcal{E}$-quasi-continuous $m_{1-}$ version. We will denote them by $\tilde{u}$.

\subsection{Dirichlet forms and Markov processes}

Let $\Delta$ be adjoint to $E^{1}$ as the point at infinity. We adopt the convention that every function $f$ on $E^{1}$ (resp. $E_{0, T}$ ) is extended to $E^{1} \cup\{\Delta\}$ (resp. $E_{0, T} \cup\{\Delta\}$ ) by setting $f(\Delta)=0$.

Let $\Omega_{1}=\left\{\omega:[0, \infty) \rightarrow E^{1} \cup\{\Delta\}: \omega(s)=\Delta, s \geq t\right.$ if $\left.\omega(t)=\Delta\right\}$ and

$$
X^{1}: \Omega_{1} \rightarrow \Omega_{1}, \quad X_{t}^{1}(\omega)=\omega(t)
$$

By [20, Theorem 4.2] (see also [22, Theorem 5.1] or [23, Theorem 6.3.1]), there exists a Hunt process $\mathbb{X}^{1}=\left(\Omega_{1},\left(\mathcal{F}_{t}^{1}\right)_{t \geq 0},\left(X_{t}^{1}\right)_{t \geq 0},\left(P_{x}^{1}\right)_{x \in E^{1} \cup\{\Delta\}}\right)$ with life time $\zeta^{1}$ and cemetery state $\Delta$ associated with $\mathcal{E}^{1}$ in the resolvent sense, i.e. for all $\alpha>0$ and $f \in \mathcal{B}_{b}\left(E^{1}\right) \cap L^{2}\left(E^{1} ; m_{1}\right)$ the resolvent of $\mathbb{X}^{1}$ defined as

$$
R_{\alpha}^{1} f(x)=E_{x}^{1} \int_{0}^{\infty} e^{-\alpha t} f\left(X_{t}^{1}\right) \mathrm{d} t, \quad x \in E^{1}, \quad f \in \mathcal{B}_{b}\left(E^{1}\right)
$$


( $E_{x}^{1}$ stands for the expectation with respect to $P_{x}^{1}$ ) is an $\mathcal{E}^{1}$-quasi-continuous $m_{1}$ version of the resolvent $G_{\alpha}^{1} f$. Let

$$
v: \Omega_{1} \rightarrow \Omega_{1}, \quad(v(\omega))(t)=\pi(\omega(0))+t,
$$

where $\pi: E^{1} \rightarrow \mathbb{R}$ is the projection on $\mathbb{R}$. Note that under the measure $P_{x}$ with $x=\left(s, x^{0}\right) \in E^{1}, v$ is the uniform motion to the right, i.e. $v(t)=v(0)+t, v(0)=s$. By [20, Theorem 4.2] (or [22, Theorem 5.1]),

$$
X_{t}^{1}=\left(v(t), X_{v(t)}^{0}\right), \quad t \geq 0
$$

under the measure $P_{x}$ for every $x \in E^{1}$.

Recall that $B \in \mathcal{B}\left(E^{1}\right)$ is called $\mathbb{X}^{1}$-exceptional if $P_{x}^{1}\left(\sigma_{B}<\infty\right)=0$ for $m_{1}$-a.e. $x \in E^{1}$, where

$$
\sigma_{B}=\inf \left\{t>0: X_{t}^{1} \in B\right\}
$$

By the remarks in [22, page 298] (see also [21, Lemma 2.3]),

$$
B \text { is } \mathcal{E}^{1} \text {-exceptional if and only if } B \text { is } \mathbb{X}^{1} \text {-exceptional. }
$$

Similarly, by [31, Sect. IV.2], there exists a Hunt process $\mathbb{X}=\left(\Omega,\left(\mathcal{F}_{t}\right)_{t \geq 0},\left(X_{t}\right)_{t \geq 0}\right.$, $\left(P_{x}\right)_{x \in E_{0, T} \cup\{\Delta\}}$ ) with life time $\zeta$ associated with $\mathcal{E}$ in the resolvent sense, i.e. for all $\alpha>0$ and $f \in \mathcal{B}_{b}\left(E_{0, T}\right) \cap L^{2}\left(E_{0, T} ; m_{1}\right)$,

$$
R_{\alpha} f(x)=E_{x} \int_{0}^{\infty} e^{-\alpha t} f\left(X_{t}\right) \mathrm{d} t, \quad x \in E_{0, T}, \quad f \in \mathcal{B}_{b}\left(E_{0, T}\right)
$$

( $E_{x}$ stands for the expectation with respect to $P_{x}$ ) is an $\mathcal{E}$-quasi-continuous $m_{1}$-version of the resolvent $G_{\alpha} f$. In this case $\Omega=\left\{\omega:[0, \infty) \rightarrow E_{0, T} \cup\{\Delta\}: \omega(t)=\Delta, s \geq t\right.$ if $\omega(s)=\Delta\}$ and

$$
X: \Omega \rightarrow \Omega, \quad X_{t}(\omega)=\omega(t) .
$$

It is clear that $\Omega \subset \Omega_{1}$ and $X=X^{1}$ on $\Omega$.

A set $B \in \mathcal{B}\left(E_{0, T}\right)$ is called $\mathbb{X}$-exceptional if $P_{x}\left(\sigma_{B}<\infty\right)=0$ for $m_{1}$-a.e. $x \in E_{0, T}$, where $\sigma_{B}$ is defined as before but with $\mathbb{X}^{1}$ replaced by $\mathbb{X}$. By [31, Theorem IV.3.8],

$B$ is $\mathcal{E}$-exceptional if and only if $B$ is $\mathbb{X}$-exceptional.

Let $f \in \mathcal{B}\left(E_{0, T}\right) \cap L^{2}\left(E_{0, T} ; m_{1}\right), g \in \mathcal{B}\left(E^{1}\right) \cap L^{2}\left(E^{1} ; m_{1}\right)$ and $v=R_{\alpha} f$, $u=R_{\alpha}^{1} g$. Since $v$ is an $m_{1}$ version of $G_{\alpha} f$ and $u$ is an $m_{1}$-version of $G_{\alpha}^{1} g$, we have $v \in \mathcal{W}_{T}, u \in \mathcal{W}^{1}$ and

$$
\mathcal{E}_{\alpha}(v, \eta)=(f, \eta)_{L^{2}\left(E_{0, T} ; m_{1}\right)}, \quad \eta \in \mathcal{W}_{0}, \quad \mathcal{E}_{\alpha}^{1}(u, \eta)=(g, \eta)_{L^{2}\left(E^{1} ; m_{1}\right)}, \quad \eta \in \mathcal{W}^{1}
$$


Let $g=f$ on $E_{0, T}$ and $g=0$ on $E^{1} \backslash E_{0, T}$. Then, by (2.5) and (2.6), $u_{\mid E_{0, T}} \in \mathcal{W}_{T}$. So, in fact $u_{\mid E_{0, T}}$ is a solution to the first equation in (2.10). By uniqueness of a solution to (2.10), we get $v=u_{\mid E_{0, T}}$. By this and (2.5) and (2.8), we obtain

$$
E_{x}^{1} \int_{0}^{\infty} e^{-\alpha t} f\left(X_{t}^{1}\right) \mathrm{d} t=E_{x} \int_{0}^{\infty} e^{-\alpha t} f\left(X_{t}\right) \mathrm{d} t
$$

for every $\alpha \geq 0$ and every $f \in \mathcal{B}^{+}\left(E^{1}\right)$ such that $\mathbf{1}_{E_{0, T}} f=f$. Let $\Pi: \Omega_{1} \rightarrow \Omega$ be defined as

$$
\Pi(\omega)(t)=\omega(t), \quad t<T-v(0), \quad \Pi(\omega)(t)=\Delta, \quad t \geq T-v(0) .
$$

Observe that

$$
E_{x}^{1} \int_{0}^{\infty} e^{-\alpha t} f\left(X_{t}^{1}\right) \mathrm{d} t=\int_{\Omega} \int_{0}^{\infty} e^{-\alpha t} f\left(X_{t}\right) \mathrm{d} t \mathrm{~d}\left(P_{x}^{1} \circ \Pi^{-1}\right) .
$$

Hence

$$
P_{x}=P_{x}^{1} \circ \Pi^{-1}, \quad x \in E_{0, T} .
$$

Therefore, for every $x \in E_{0, T}$,

$$
\begin{aligned}
& P_{x}(\zeta \leq T-v(0))=P_{x}\left(\omega \in \Omega: \inf \left\{t \geq 0 ; X_{t}(\omega) \notin E_{0, T}\right\} \leq T-v(0)(\omega)\right) \\
& =P_{x}\left(\omega \in \Omega ; \inf \left\{t \geq 0: X_{t}^{1}(\omega) \notin E_{0, T}\right\} \leq T-v(0)(\omega)\right) \\
& =\left(P_{x}^{1} \circ \Pi^{-1}\right)\left(\omega \in \Omega: \inf \left\{t \geq 0 ; X_{t}^{1}(\omega) \notin E_{0, T}\right\} \leq T-v(0)(\omega)\right) \\
& =P_{x}^{1}\left(\omega \in \Omega_{1}: \inf \left\{t \geq 0 ; X_{t}^{1}(\Pi(\omega)) \notin E_{0, T}\right\} \leq T-v(0)(\Pi(\omega))\right) \\
& =P_{x}^{1}\left(\omega \in \Omega_{1}: \inf \left\{t \geq 0:\left(v(t)(\Pi(\omega)), X_{v(t)(\Pi(\omega))}^{0}(\Pi(\omega))\right) \notin E_{0, T}\right\}\right. \\
& \quad \leq T-v(0)(\Pi(\omega))) \\
& \geq P_{x}^{1}\left(\omega \in \Omega_{1}: \inf \{t \geq 0: v(t)(\Pi(\omega)) \notin(0, T)\} \leq T-v(0)(\Pi(\omega))\right)=1
\end{aligned}
$$

Thus

$$
\zeta=\zeta \wedge(T-v(0)) \quad P_{x} \text {-a.s. }
$$

In this paper, we denote by $\hat{\mathbb{X}}^{1}=\left(\Omega_{1},\left(\hat{\mathcal{F}}_{t}^{1}\right)_{t \geq 0},\left(X_{t}^{1}\right)_{t \geq 0},\left(\hat{P}_{x}^{1}\right)_{x \in E^{1} \cup\{\Delta\}}, \zeta^{1}\right)$ the dual process of $\mathbb{X}^{1}$, i.e. a Hunt process whose resolvents $\hat{R}_{\alpha}^{1} f$ are $\mathcal{E}^{1}$-quasi-continuous $m_{1}$-versions of $\hat{G}_{\alpha}^{1} f$ for any $f \in \mathcal{B}_{b}\left(E^{1}\right) \cap L^{2}\left(E^{1} ; m_{1}\right)$. By [22, Theorem 5.1],

$$
X_{t}^{1}=\left(\hat{v}(t), \hat{X}_{\hat{v}(t)}^{0}\right), \quad t \geq 0
$$

where $\hat{v}$ is the uniform motion to the left, i.e. $\hat{v}(t)=\hat{v}(0)-t, \hat{v}(0)=s$ under the measure $\hat{P}_{x}$ with $x=\left(s, x^{0}\right) \in E^{1}$. 
We denote by $\hat{\mathbb{X}}=\left(\Omega,\left(\hat{\mathcal{F}}_{t}\right)_{t \geq 0},\left(X_{t}\right)_{t \geq 0},\left(\hat{P}_{x}\right)_{x \in E_{0, T} \cup\{\Delta\}}, \zeta\right)$ the dual process of $\mathbb{X}$. It is a Hunt process associated with the dual form $\hat{\mathcal{E}}$, For all $\alpha>0$ and $f \in$ $\mathcal{B}_{b}\left(E_{0, T}\right) \cap L^{2}\left(E_{0, T} ; m_{1}\right)$ the resolvent of $\hat{\mathbb{X}}$ defined as

$$
\hat{R}_{\alpha} f(x)=\hat{E}_{x} \int_{0}^{\infty} e^{-\alpha t} f\left(X_{t}\right) \mathrm{d} t, \quad x \in E_{0, T},
$$

where $\hat{E}_{x}$ denotes the expectation with respect to $\hat{P}_{x}$, is an $\mathcal{E}$-quasi-continuous $m_{1}$ version of the coresolvent $\hat{G}_{\alpha} f$. A modification of the argument used to prove (2.12) and (2.13) shows that

$$
\hat{P}_{x}=\hat{P}_{x}^{1} \circ \hat{\Pi}^{-1}, \quad \zeta=\zeta \wedge \hat{v}(0), \quad \hat{P}_{x} \text {-a.s. }
$$

for $x \in E_{0, T}$, where $\hat{\Pi}: \Omega_{1} \rightarrow \Omega$ is defined as

$$
\hat{\Pi}(\omega)(t)=\omega(t), \quad t<\hat{v}(0), \quad \hat{\Pi}(\omega)(t)=\Delta, \quad t \geq \hat{v}(0) .
$$

The relation (2.13) [resp. (2.15)] implies that the operator $G_{\alpha}$ (resp. $\hat{G}_{\alpha}$ ) is well defined for $\alpha=0$, and that $R_{0} f$ (resp. $\hat{R} f$ ) is $\mathcal{E}$-quasi-continuous $m_{1}$-version of $G_{0} f$ (resp. $\hat{G}_{0} f$ ) for every $f \in \mathcal{H}$, Indeed, by (2.13), for every positive $f \in \mathcal{B}\left(E_{0, T}\right)$,

$$
R f \leq e^{\alpha T} R_{\alpha} f
$$

(we write $R$ instead of $R_{0}$ ). Hence, for every $f \in \mathcal{H} \cap \mathcal{B}\left(E_{0, T}\right), R f \in \mathcal{H}$. By the resolvent identity,

$$
R f=R_{\alpha} f+\alpha R_{\alpha} R f
$$

Therefore $R f \in G_{\alpha}(\mathcal{H})$, and by (2.4),

$$
\mathcal{E}(R f, g)=(f, g)_{\mathcal{H}}, \quad f \in \mathcal{H}, g \in \mathcal{V} \text {. }
$$

In other words, $G_{0} f=R f m_{1}$-a.e. Of course, $R f$ is $\mathcal{E}$-quasi-continuous since $R f=$ $R_{\alpha}(f+\alpha R f)$. A similar argument applies to $\hat{R}_{0}$ and $\hat{G}_{0}$.

\subsection{Smooth measures}

A Borel (signed) measure $\mu$ on $E_{0, T}$ is called $\mathcal{E}$-smooth if it does not charge exceptional sets, i.e. for any Borel set $B \subset E_{0, T}$, if $\operatorname{Cap}_{\psi}(B)=0$, then $\mu(B)=0$, and there exists an $\mathcal{E}$-nest $\left\{F_{n}\right\}$ of compact subsets of $E_{0, T}$ such that $|\mu|\left(F_{n}\right)<\infty$ for $n \in \mathbb{N}$, where $|\mu|$ denotes the variation of $\mu$. A Borel measure $\mu$ on $E^{1}$ is called $\mathcal{E}^{1}$-smooth if it does not charge $\mathcal{E}^{1}$-exceptional sets and there exists a generalized $\mathcal{E}^{1}$-nest $\left\{F_{n}\right\}$ such that $|\mu|\left(F_{n}\right)<\infty$ for $n \in \mathbb{N}$.

The set of all $\mathcal{E}$-smooth (resp. $\mathcal{E}^{1}$-smooth) measures will be denoted by $S\left(E_{0, T}\right)$ (resp. $\left.S^{1}\left(E^{1}\right)\right) . \mathcal{M}_{b}\left(E_{0, T}\right)\left(\right.$ resp. $\left.\mathcal{M}_{b}^{1}\left(E^{1}\right)\right)$ denotes the set of all bounded Borel measures on $E_{0, T}$ (resp. $E^{1}$ ), i.e. Borel measures $\mu$ such that $|\mu|\left(E_{0, T}\right)<\infty$ (resp. 
$\left.|\mu|\left(E^{1}\right)<\infty\right) . \mathcal{M}_{0, b}\left(E_{0, T}\right)\left(\right.$ resp. $\left.\mathcal{M}_{0, b}^{1}\left(E^{1}\right)\right)$ denotes the subset of $\mathcal{M}_{b}\left(E_{0, T}\right)$ (resp. $\left.\mathcal{M}_{b}^{1}\left(E^{1}\right)\right)$ consisting of all $\mathcal{E}$-smooth (resp. $\mathcal{E}^{1}$-smooth) measures.

Let $\mu$ be a positive Borel measure on $E_{0, T}$ such that $\mu$ does not charge sets of zero capacity. We call it a measure of finite energy integral if there is $C \geq 0$ such that for every positive $\eta \in \mathcal{W}_{0}$,

$$
\int_{E_{0, T}} \tilde{\eta} \mathrm{d} \mu \leq C\|\eta\|_{\mathcal{W}}
$$

If there is $C \geq 0$ such that for every positive $\eta \in \mathcal{W}_{T}$,

$$
\int_{E_{T}} \tilde{\eta} \mathrm{d} \mu \leq C\|\eta\|_{\mathcal{W}}
$$

we call it a measure of finite co-energy integral. In both cases, $\tilde{\eta}$ denotes an $\mathcal{E}$-quasicontinuous $m_{1}$-version of $\eta$. The set of all positive smooth measures on $E_{0, T}$ of finite energy (resp. co-energy) integral will be denoted by $S_{0}\left(E_{0, T}\right)\left(\operatorname{resp} . \hat{S}_{0}\left(E_{0, T}\right)\right)$.

LEMMA 2.1. Let $\mu$ be a positive Borel measure on $E_{0, T}$. Then

(i) $\mu \in S_{0}\left(E_{0, T}\right)$ if and only if there exists $u \in \mathcal{V}$ such that

$$
\int_{E_{0, T}} \tilde{v} \mathrm{~d} \mu=\mathcal{E}_{1}(u, v), \quad v \in \mathcal{W}_{0} .
$$

(ii) $\mu \in \hat{S}_{0}\left(E_{0, T}\right)$ if and only if there exists $u \in \mathcal{V}$ such that

$$
\int_{E_{0, T}} \tilde{v} \mathrm{~d} \mu=\mathcal{E}_{1}(v, u), \quad v \in \mathcal{W}_{T} .
$$

Proof. We provide the proof of (i). The proof of (ii) is analogous. If (2.18) is satisfied, then of course $\mu \in S_{0}\left(E_{0, T}\right)$. Suppose that $\mu$ is of finite energy integral. Then, by [33, Lemma 4.2] applied to the dual form $\hat{\mathcal{E}}$, for every positive $\eta \in \mathcal{W}_{0}$ there exists $u \in$ such that

$$
\int_{E_{0, T}} \alpha \widetilde{\alpha \hat{G}_{\alpha} \eta} \mathrm{d} \mu=\mathcal{E}_{1}\left(u, \alpha \hat{G}_{\alpha} \eta\right)
$$

where $\widetilde{\hat{G}_{\alpha} \eta}$ is a quasi-continuous modification of $\hat{G}_{\alpha} \eta$. By [30, Proposition 2.7], $\alpha \hat{G}_{\alpha} \eta \rightarrow \eta$ strongly in $\mathcal{W}_{0}$. Therefore letting $\alpha \rightarrow \infty$ in (2.20) and applying [31, Corollary III.3.8], Fatou's lemma and [28, Lemma 2], we get (2.17).

Let $\mu \in S_{0}\left(E_{0, T}\right)$. The element $u \in \mathcal{V}$ defined by (2.18) is uniquely determined. We will denote it by $U_{1} \mu$. Similarly, for $\mu \in \hat{S}_{0}\left(E_{0, T}\right)$, the element $u \in \mathcal{V}$ defined by (2.19) is uniquely determined. We will denote it by $\hat{U}_{1} \mu$. We also set

$$
U_{\alpha} \mu=U_{1} \mu+(1-\alpha) G_{\alpha} U_{1} \mu, \quad \hat{U}_{\alpha} \mu=\hat{U}_{1} \mu+(1-\alpha) \hat{G}_{\alpha} \hat{U}_{1} \mu \quad \alpha \geq 0 .
$$

It is clear that for any $\alpha \geq 0$,

$$
\int_{E_{0, T}} \tilde{v} \mathrm{~d} \mu=\mathcal{E}_{\alpha}\left(U_{\alpha} \mu, v\right), v \in \mathcal{W}_{0}, \quad \int_{E_{0, T}} \tilde{v} \mathrm{~d} \mu=\mathcal{E}_{\alpha}\left(v, \hat{U}_{\alpha} \mu\right), v \in \mathcal{W}_{T} .
$$




\section{Parabolic capacity}

Our basic capacity associated with $\mathcal{E}$ is $\mathrm{Cap}_{\psi}$. Exceptional sets with respect to $\mathrm{Cap}_{\psi}$ have nice probabilistic interpretation given by (2.9). However, in the literature devoted to partial differential equations, usually some other notions of capacity are used. In this section, we recall some of them and prove that they are all equivalent to $\mathrm{Cap}_{\psi}$. These results will be needed in the next sections.

LEMMA 3.1. For any $A \subset E_{0, T}, \operatorname{Cap}_{\psi}(A)=0$ if and only if $\operatorname{Cap}_{\mid E_{0, T}}^{1}(A)=0$.

Proof. Since both $\mathrm{Cap}_{\psi}$ and $\mathrm{Cap}_{\mid E_{0, T}}^{1}$ are Choquet capacities, it is enough to prove that for any $B \in \mathcal{B}\left(E_{0, T}\right), \operatorname{Cap}_{\psi}(B)=0$ if and only if $\operatorname{Cap}^{1}(B)=0$. Suppose that $\operatorname{Cap}^{1}(B)=0$. Then, by (2.7), $P_{x}^{1}\left(\sigma_{B}<\infty\right)=0$ for $m_{1}$-a.e. $x \in E^{1}$. Observe that

$$
\left\{\omega \in \Omega_{1} ; \exists_{t>0} X_{t}^{1}(\omega) \in B\right\}=\Pi^{-1}\left(\left\{\omega \in \Omega ; \exists_{t>0} X_{t}(\omega) \in B\right\}\right) .
$$

Hence, by (2.12), $P_{x}\left(\sigma_{B}<\infty\right)=0$ for $m_{1}$-a.e. $x \in E_{0, T}$. Consequently, $\operatorname{Cap}_{\psi}(B)=$ 0 by (2.9). Now suppose that $\operatorname{Cap}_{\psi}(B)=0$. Then, by [31, Lemma III.2.9] and (2.9), $P_{x}\left(\sigma_{B}<\infty\right)=\hat{P}_{x}\left(\sigma_{B}<\infty\right)=0$ for $m_{1}$-a.e. $x \in E_{0, T}$. From this and (2.6) and (2.12) it follows that

$$
P_{x}^{1}\left(\sigma_{B}<\infty\right)=0 \text { for } m_{1} \text {-a.e. } x \in[0, \infty) \times E .
$$

Similarly, from (2.14) and (2.15) it follows that

$$
\hat{P}_{x}^{1}\left(\sigma_{B}<\infty\right)=0 \text { for } m_{1} \text {-a.e. } x \in(-\infty, T] \times E .
$$

By [31, Lemma III.2.9], for every $\eta \in \mathcal{B}_{b}^{+}\left(E^{1}\right)$,

$$
E_{\eta \cdot m_{1}}^{1} e^{-\sigma_{B}} \int_{\sigma_{B}}^{\infty} e^{-t} \eta\left(X_{t}^{1}\right) \mathrm{d} t=\hat{E}_{\eta \cdot m_{1}}^{1} e^{-\sigma_{B}} \int_{\sigma_{B}}^{\infty} e^{-t} \eta\left(X_{t}^{1}\right) \mathrm{d} t
$$

where $E_{\eta \cdot m_{1}}^{1}$ denotes the expectation with respect to the measure $P_{\eta \cdot m_{1}}^{1}$ defined as $P_{\eta \cdot m_{1}}(\cdot)=\int_{E^{1}} P_{x}(\cdot) \eta(x) m_{1}(\mathrm{~d} x)$ and $\hat{E}_{\eta \cdot m_{1}}^{1}$ denotes the expectation with respect to $\hat{P}_{\eta \cdot m_{1}}^{1}$ defined as $P_{\eta \cdot m_{1}}^{1}$ but with $P_{x}$ replaced by $\hat{P}_{x}$. Let $\eta=0$ on $[T, \infty) \times E$ and $\eta>0$ on $(-\infty, T) \times E$. Then, by (3.2), the right-hand side of (3.3) equals zero, which implies that $P_{x}^{1}\left(\sigma_{B}<\infty\right)=0$ for $m_{1}$-a.e. $x \in(-\infty, T] \times E$. This when combined with (3.1) shows that $P_{x}^{1}\left(\sigma_{B}<\infty\right)=0$ for $m_{1}$-a.e. $x \in E^{1}$. By (2.7), $\operatorname{Cap}^{1}(B)=0$.

We now recall the capacity considered in Pierre [28] (see also [26,27]). Let $U$ be a relatively compact open subset of $E_{0, T}$. By [31, Proposition III.1.6], for every $n \geq 1$ there exists a unique solution $e_{U}^{n} \in \mathcal{W}_{T}$ of the following problem

$$
\mathcal{E}_{1}\left(e_{U}^{n}, \eta\right)=n\left(\left(e_{U}^{n}-\mathbf{1}_{U}\right)^{-}, \eta\right), \quad \eta \in \mathcal{V} .
$$


By [31, Proposition III.1.7], $\left\{e_{U}^{n}\right\}$ converges strongly in $\mathcal{H}$ and weakly in $\mathcal{V}$. Set $e_{U}=\lim _{n \rightarrow \infty} e_{U}^{n}$. By [31, Proposition III.1.7], for every $\eta \in \mathcal{W}_{0}$ such that $\eta \geq \mathbf{1}_{U}$ $m_{1}$-a.e. we have

$$
\mathcal{E}_{1}\left(e_{U}, \eta\right) \geq \mathcal{B}_{1}\left(e_{U}, e_{U}\right)
$$

By Lemma 2.1 (see also [28]), there exists a measure $\mu_{U} \in S_{0}\left(E_{0, T}\right)$ such that $e_{U}=U_{1} \mu_{U}$. By [27, Proposition II.1], $\operatorname{supp}\left[\mu_{U}\right] \subset \bar{U}$. In [28], the capacity of $U$ is defined by

$$
c_{0}(U)=\mu_{U}\left(E_{0, T}\right)
$$

As usual, for an arbitrary $A \subset E_{0, T}$, we define

$$
c_{0}(A)=\inf \left\{c_{0}(U), A \subset U, U \text { open }\right\} .
$$

By [28, Proposition 2], $c_{0}$ is a Choquet capacity.

LEMMA 3.2. For any $A \subset E_{0, T}, \operatorname{Cap}_{\psi}(A)=0$ if and only if $c_{0}(A)=0$.

Proof. Let $U$ be a relatively compact open subset of $E_{0, T}$. Let $\widetilde{\hat{G}_{1} \psi}$ be a quasicontinuous modification of $\hat{G}_{1} \psi$. By the definitions of $\mathrm{Cap}_{\psi}$ and $U_{1} \mu_{U}$, and the fact that $\hat{G}_{1}$ is Markovian, we have

$\operatorname{Cap}_{\psi}(U) \leq \int_{E_{0, T}} e_{U} \psi \mathrm{d} m_{1}=\mathcal{E}_{1}\left(e_{U}, \hat{G}_{1} \psi\right)=\int_{E_{0, T}} \widetilde{\hat{G}_{1} \psi} \mathrm{d} \mu_{U} \leq \mu_{U}\left(E_{0, T}\right)=c_{0}(U)$.

Hence $\mathrm{Cap}_{\psi} \leq c_{0}$. Let $K$ be a compact subset of $E_{0, T}$ such that $\operatorname{Cap}_{\psi}(K)=0$. Then there exists a nonincreasing sequence $U_{n}$ of open relatively compact subsets of $E_{0, T}$ such that $\operatorname{Cap}_{\psi}\left(U_{n}\right) \searrow 0$ and $K \subset U_{n}$. Let $\eta \in \mathcal{W}_{0} \cap C_{c}\left(E_{0, T}\right)$ be such that $\eta \geq \mathbf{1}_{U_{1}}$ (see [27, Lemma II.3]). Then, by (3.4),

$$
\mathcal{B}_{1}\left(e_{U_{n}}, e_{U_{n}}\right) \leq \mathcal{E}_{1}\left(e_{U_{n}}, \eta\right)=\int_{E_{0, T}} \tilde{\eta} \mathrm{d} \mu_{U_{n}}=c_{0}\left(U_{n}\right) .
$$

Since $\left\{U_{n}\right\}$ is a nonincreasing, $\left\{e_{U_{n}}\right\}$ is nonincreasing. By [31, Proposition IV.3.4], $e_{U_{n}} \searrow 0 m_{1}$-a.e. By (3.5),

$$
\mathcal{B}_{1}\left(e_{U_{n}}, e_{U_{n}}\right) \leq c_{0}\left(U_{1}\right)
$$

Therefore there exists a subsequence (still denoted by $n$ ) such that $\left\{e_{U_{n}}\right\}$ is weakly convergent in $\mathcal{V}$. Since $e_{U_{n}} \searrow 0 m_{1}$-a.e., in fact $e_{U_{n}} \rightarrow 0$ weakly in $\mathcal{V}$. Thus $\mathcal{E}_{1}\left(e_{U_{n}}, \eta\right) \rightarrow 0, \eta \in \mathcal{W}_{0}$. By (3.5),

$$
\mathcal{E}_{1}\left(e_{U_{n}}, \eta\right) \geq c_{0}(K)
$$

Hence $c_{0}(K)=0$. Since $\mathrm{Cap}_{\psi}$ and $c_{0}$ are Choquet capacities, this implies the desired result. 
Write

$$
\mathcal{W}^{2}=\left\{u \in \mathcal{W}: \partial_{t} u \in L^{2}\left(E_{0, T} ; m_{1}\right)\right\}, \quad \mathcal{C}=C_{c}\left(E_{0, T}\right) \cap \mathcal{W}^{2},
$$

where $C_{c}\left(E_{0, T}\right)$ is the set of all real continuous functions on $E_{0, T}$ having compact support. For a compact set $K \subset E_{0, T}$, we set

$$
\operatorname{CAP}(K)=\inf \left\{\|u\|_{\mathcal{W}}: u \in \mathcal{C}, u \geq \mathbf{1}_{K}\right\} .
$$

Next, for an open $U \subset E_{0, T}$, we set $\mathrm{CAP}(U)=\sup \{\mathrm{CAP}(K): K \subset U, K$-compact $\}$, and finally, for arbitrary $A \subset E_{0, T}$, we set $\operatorname{CAP}(A)=\inf \{\mathrm{CAP}(U): A \subset U, U$ open $\}$. We will also consider the capacity $c_{2}$ (see [28]) defined as

$$
\mathrm{c}_{2}(U)=\inf \left\{\|u\|_{\mathcal{W}}: u \in \mathcal{W}, u \geq \mathbf{1}_{U} m_{1} \text {-a.e. }\right\}
$$

for an open set $U \subset E_{0, T}$, and then by $c_{2}(A)=\inf \left\{\mathrm{c}_{2}(U): A \subset U, U\right.$ open $\}$ for an arbitrary set $A \subset E_{0, T}$. By [28, Proposition 2], $\mathrm{c}_{2}$ is a Choquet capacity.

LEMMA 3.3. For any $A \subset E_{0, T}, \mathrm{c}_{2}(A)=0$ if and only if $\mathrm{Cap}_{\psi}(A)=0$.

Proof. Follows from Lemma 3.2 because by [28, Theorem 1], for any $A \subset E_{0, T}$, $\mathrm{c}_{2}(A)=0$ if and only if $c_{0}(A)=0$.

LEMMA 3.4. For any $A \subset E_{0, T}, \operatorname{CAP}(A)=0$ if and only if $\operatorname{Cap}_{\psi}(A)=0$.

Proof. By virtue of Lemma 3.3, it suffices to prove that CAP is equivalent to $\mathrm{c}_{2}$. Let $K$ be a compact subset of $E_{0, T}$ and let $\varepsilon>0, a>1$. Choose $\eta_{\varepsilon} \in \mathcal{C}$ such that $\left\|\eta_{\varepsilon}\right\|_{\mathcal{W}} \leq \mathrm{CAP}(K)+\varepsilon$ and $\eta_{\varepsilon} \geq \mathbf{1}_{K}$. Since $\eta_{\varepsilon}$ is continuous, there exists an open set $V_{a}$ such that $a \eta_{\varepsilon} \geq \mathbf{1}_{V_{a}}$ and $K \subset V_{a}$. We have

$$
\mathrm{c}_{2}(K) \leq \mathrm{c}_{2}\left(V_{a}\right) \leq\left\|a \eta_{\varepsilon}\right\| \mathcal{W} \leq a(\operatorname{CAP}(K)+\varepsilon) .
$$

Letting $\varepsilon \downarrow 0$ and then $a \downarrow 1$ we see that $\mathrm{c}_{2}(K) \leq \mathrm{CAP}(K)$ for every compact $K \subset E_{0, T}$. From this and the fact that $\mathrm{c}_{2}$ is a Choquet capacity we conclude that $\mathrm{c}_{2} \leq \mathrm{CAP}$.

Now suppose that $\mathrm{c}_{2}(K)=0$ for some compact set $K \subset E_{0, T}$. Let $U_{n}=\{(s, x) \in$ $\left.E_{0, T}: \operatorname{dist}((s, x), K)<n^{-1}\right\}$. Since $E_{0, T}$ is locally compact and $K$ is compact, we may assume that $U_{n}$ are relatively compact. Since $c_{2}$ is a Choquet capacity, $b_{n}:=$ $\mathrm{c}_{2}\left(U_{n}\right) \searrow 0$. Fix $\varepsilon>0$ and choose $\eta_{n}^{\varepsilon} \in \mathcal{W}$ such that $\eta_{n}^{\varepsilon} \geq \mathbf{1}_{U_{n}} m_{1}$-a.e. and $\left\|\eta_{n}^{\varepsilon}\right\| \mathcal{W} \leq b_{n}+\varepsilon$. By putting $\eta_{n}^{\varepsilon}(t)=\eta_{n}^{\varepsilon}(T)$ for $t \geq T$ and $\eta_{n}^{\varepsilon}(t)=\eta_{n}^{\varepsilon}(0)$ for $t \leq 0$, we may assume that $\eta_{n}^{\varepsilon} \in \mathcal{W}^{1}$. Write

$$
J_{n}\left(\eta_{n}^{\varepsilon}\right)=2 n \int_{0}^{1 /(2 n)} \eta_{n}^{\varepsilon}(t+s) j(2 n s) \mathrm{d} s,
$$

where $j$ is a smooth positive function with support in $[0,1]$ such that $\int_{\mathbb{R}} j(t) \mathrm{d} t=1$. It is clear that $J_{n}\left(\eta_{n}^{\varepsilon}\right) \in \mathcal{W}^{2},\left\|J_{n}\left(\eta_{n}^{\varepsilon}\right)\right\| \mathcal{W} \leq\left\|\eta_{n}^{\varepsilon}\right\|_{\mathcal{W}}$ and

$$
J_{n}\left(\eta_{n}^{\varepsilon}\right) \geq \mathbf{1}_{U_{n-1}} \quad m_{1} \text {-a.e. }
$$


By regularity of the forms $B^{(t)}$, for every $a>0$ there exists $\eta_{n}^{\varepsilon, a} \in \mathcal{C}$ such that

$$
\left\|J_{n}\left(\eta_{n}^{\varepsilon}\right)-\eta_{n}^{\varepsilon, a}\right\|_{\mathcal{W}^{2}} \leq a
$$

(see [22, Lemma 1.1]). Let $\xi_{n} \in \mathcal{C}$ be a positive function such that $\xi_{n}=1$ on $K$ and $\operatorname{supp}\left[\xi_{n}\right] \subset U_{n-1}$. The existence of $\xi_{n}$ follows from [22, Lemma 1.1]. We put

$$
\bar{\eta}_{n}^{\varepsilon, a}=\eta_{n}^{\varepsilon, a}+\left(\xi_{n}-\eta_{n}^{\varepsilon, a}\right)^{+}
$$

Observe that $\bar{\eta}_{n}^{\varepsilon, a} \in \mathcal{C}$ and $\bar{\eta}_{n}^{\varepsilon, a} \geq \mathbf{1}_{K}$. Let $a_{l} \downarrow 0$. By (3.7), up to a subsequence, $\left(\xi_{n}-\eta_{n}^{\varepsilon, a_{l}}\right)^{+} \rightarrow\left(\xi_{n}-J_{n}\left(\eta_{n}^{\varepsilon}\right)\right)^{+}$weakly in $\mathcal{W}^{2}$ as $l \rightarrow \infty$. Hence, up to a subsequence,

$$
\frac{1}{k} \sum_{l=1}^{k}\left(\xi_{n}-\eta_{n}^{\varepsilon, a_{l}}\right)^{+} \rightarrow\left(\xi_{n}-J_{n}\left(\eta_{n}^{\varepsilon}\right)\right)^{+}
$$

strongly in $\mathcal{W}^{2}$. Furthermore,

$$
\mathrm{CAP}(K) \leq\left\|\frac{1}{k} \sum_{l=1}^{k} \bar{\eta}_{n}^{\varepsilon, a_{l}}\right\|_{\mathcal{W}} \leq\left\|\frac{1}{k} \sum_{l=1}^{k} \eta_{n}^{\varepsilon, a_{l}}\right\|_{\mathcal{W}}+\left\|\frac{1}{k} \sum_{l=1}^{k}\left(\xi_{n}-\eta_{n}^{\varepsilon, a_{l}}\right)^{+}\right\|_{\mathcal{W}^{2}} .
$$

By (3.6), $\left(\xi_{n}-J_{n}\left(\eta_{n}^{\varepsilon}\right)\right)^{+}=0$, so letting $k \rightarrow \infty$ in the above inequality and using (3.7) and (3.8) we get

$$
\mathrm{CAP}(K) \leq\left\|J_{n}\left(\eta_{n}^{\varepsilon}\right)\right\|_{\mathcal{W}} \leq\left\|\eta_{n}^{\varepsilon}\right\|_{\mathcal{W}} \leq b_{n}+\varepsilon .
$$

Hence $\operatorname{CAP}(K)=0$. Since $\mathrm{c}_{2}$ is a Choquet capacity, this implies that $\operatorname{CAP}(A)=0$ if $\mathrm{c}_{2}(A)=0$.

\section{Smooth measures and associated additive functionals}

The results of this section will be needed in Sects. 8 and 9. Recall that a positive additive functional (AF in abbreviation) $A^{1}$ of $\mathbb{X}^{1}$ is said to be in the Revuz correspondence with a positive $\mu \in S^{1}\left(E^{1}\right)$ if

$$
\lim _{\alpha \rightarrow \infty} \alpha E_{m_{1}}^{1} \int_{0}^{\infty} e^{-\alpha t} f\left(X_{t}^{1}\right) \mathrm{d} A_{t}^{1}=\int_{E^{1}} f(x) \mu(\mathrm{d} x), \quad f \in \mathcal{B}_{b}^{+}\left(E^{1}\right),
$$

where $E_{m_{1}}^{1}$ denotes the expectation with respect to the measure $P_{m_{1}}^{1}$ defined as $P_{m_{1}}^{1}(\cdot)=$ $\int_{E^{1}} P_{x}(\cdot) m_{1}(\mathrm{~d} x)$. Similarly, we say that a positive AF $A$ of $\mathbb{X}$ is in the Revuz correspondence with a positive $\mu \in S\left(E_{0, T}\right)$ if

$$
\lim _{\alpha \rightarrow \infty} \alpha E_{m_{1}} \int_{0}^{\infty} e^{-\alpha t} f\left(X_{t}\right) \mathrm{d} A_{t}=\int_{E_{0, T}} f(x) \mu(\mathrm{d} x), \quad f \in \mathcal{B}_{b}^{+}\left(E_{0, T}\right),
$$

where $E_{m_{1}}$ denotes the expectation with respect to $P_{m_{1}}(\cdot)=\int_{E_{0, T}} P_{x}(\cdot) m_{1}(\mathrm{~d} x)$. 
Let $A_{t}^{f}=\int_{0}^{t} f\left(X_{r}\right) \mathrm{d} A_{r}$. We have

$$
\lim _{\alpha \rightarrow \infty} \alpha^{2} E_{m_{1}} \int_{0}^{\infty} e^{-\alpha t} A_{t}^{f} \mathrm{~d} t=\lim _{\alpha \rightarrow \infty} \int_{0}^{\infty} s e^{-s} \frac{\alpha}{s} E_{m_{1}} A_{s / \alpha}^{f} \mathrm{~d} s .
$$

Since $t \mapsto E_{m_{1}} A_{t}^{f}$ is subadditive, $(1 / t) E_{m_{1}} A_{t}^{f}$ increases as $t$ decreases, and moreover, $\lim _{t \downarrow 0}(1 / t) E_{m_{1}} A_{t}^{f}=\sup _{t>0}(1 / t) E_{m} A_{t}^{f}$. Therefore, letting $\alpha \rightarrow \infty$ in (4.3), shows that

$$
\begin{aligned}
\lim _{\alpha \rightarrow \infty} \alpha E_{m_{1}} \int_{0}^{\infty} e^{-\alpha t} \mathrm{~d} A_{t}^{f} & =\lim _{\alpha \rightarrow \infty} \alpha^{2} E_{m_{1}} \int_{0}^{\infty} e^{-\alpha t} A_{t}^{f} \mathrm{~d} t \\
& =\sup _{t>0} \frac{1}{t} E_{m_{1}} A_{t}^{f} \cdot \int_{0}^{\infty} s e^{-s} \mathrm{~d} s=\sup _{t>0} \frac{1}{t} E_{m_{1}} A_{t}^{f} .
\end{aligned}
$$

A similar result holds for $A^{1}$.

It is known (see [12, Sect. 2]) that for every $\mu \in S^{1}\left(E^{1}\right)$ there exists a unique positive natural $\mathrm{AF} A^{1}$ of $\mathbb{X}^{1}$ (i.e. a positive $\mathrm{AF}$ of $\mathbb{X}^{1}$ such that $A^{1}$ and $\mathbb{X}^{1}$ have no common discontinuities) such that $A^{1}$ is in the Revuz correspondence with $\mu$. In what follows we denote it by $A^{1, \mu}$. In fact, $A^{1, \mu}$ is a predictable process (see [10, Theorem 5.3]). In the proposition below, we show a similar result for positive smooth measures on $E_{0, T}$.

PROPOSITION 4.1. Let $\mu \in S\left(E_{0, T}\right)$ be positive. There exists a unique positive natural $A F A^{\mu}$ of $\mathbb{X}$ in the Revuz correspondence with $\mu$. Moreover, for q.e. $x \in E_{0, T}$,

$$
E_{x} \int_{0}^{\infty} e^{-\alpha t} f\left(X_{t}\right) \mathrm{d} A_{t}^{\mu}=E_{x}^{1} \int_{0}^{\infty} e^{-\alpha t} f\left(X_{t}^{1}\right) \mathrm{d} A_{t}^{1, \bar{\mu}}, \quad \alpha \geq 0, \quad f \in \mathcal{B}_{b}^{+}\left(E_{0, T}\right),
$$

where $\bar{\mu}$ denotes the extension of $\mu$ to $E^{1}$ such that $\mu\left(E^{1} \backslash E_{0, T}\right)=0$ and $A^{1, \bar{\mu}}$ is the positive natural $A F$ of $\mathbb{X}^{1}$ in the Revuz correspondence with $\bar{\mu}$.

Proof. We first assume that $\mu \in S_{0}\left(E_{0, T}\right)$. Since $\mu$ is zero outside $E_{0, T}$, by Lemma 3.1 it is clear that $\bar{\mu} \in S_{0}\left(E^{1}\right)$. By the remark preceding Proposition 4.1 , there exists a positive natural AF $A^{1, \bar{\mu}}$ of $\mathbb{X}^{1}$ in the Revuz correspondence with $\bar{\mu}$. Let $R^{1} \bar{\mu}(x)=$ $E_{x}^{1} A_{\zeta}^{1, \bar{\mu}}$. By (4.1),

$$
\mathbf{1}_{E_{0, T}} R^{1} \bar{\mu}=R^{1} \bar{\mu}
$$

for $x \in E_{0, T}$. Hence, by [10, Theorem 9.3] and (2.11) (for the dual process), for every positive $\eta \in \mathcal{B}\left(E^{1}\right) \cap L^{2}\left(E^{1} ; m_{1}\right)$ such that $\eta=0$ outside $E_{0, T}$ we have

$$
\begin{aligned}
\int_{E^{1}} R^{1} \bar{\mu} \eta \mathrm{d} m_{1}=\int_{E^{1}} \hat{R}^{1} \eta \mathrm{d} \bar{\mu} & =\int_{E_{0, T}} \hat{R}^{1} \eta \mathrm{d} \bar{\mu} \\
& =\int_{E_{0, T}} \hat{R} \eta \mathrm{d} \mu=\mathcal{E}(U \mu, \hat{R} \eta)=\int_{E_{0, T}} U \mu \eta \mathrm{d} m_{1} .
\end{aligned}
$$


Thus $R^{1} \bar{\mu}=U \mu m_{1}$-a.e. on $E_{0, T}$. By (2.12) and (4.5), for all $\tau \leq \zeta$ and $x \in E_{0, T}$,

$$
E_{x}\left(R^{1} \bar{\mu}\right)\left(X_{\tau}\right)=E_{x}^{1}\left(R^{1} \bar{\mu}\right)\left(X_{\tau}^{1} \circ \Pi\right)=E_{x}^{1}\left(R^{1} \bar{\mu}\right)\left(X_{\tau}^{1}\right) \leq E_{x}^{1}\left(A_{\zeta}^{1, \bar{\mu}}-A_{\tau}^{1, \bar{\mu}}\right) .
$$

Hence $R^{1} \bar{\mu}$ is a natural potential with respect to $\mathbb{X}$. By [2, Theorem IV.4.22], there exists a unique positive natural $\mathrm{AF} A$ of $\mathbb{X}$ such that

$$
R^{1} \bar{\mu}=E . A_{\zeta} \quad \text { q.e. on } E_{0, T} .
$$

From this and the fact that $R^{1} \bar{\mu}=U \mu m_{1}$-a.e. on $E_{0, T}$ we get

$\lim _{t \rightarrow 0} \frac{1}{t} E_{\eta \cdot m_{1}} A_{t}=\left(R^{1} \bar{\mu}, \frac{1}{t}\left(\eta-T_{t} \eta\right)\right)_{\mathcal{H}}=\left(U \mu, \frac{1}{t}\left(\eta-T_{t} \eta\right)\right)_{\mathcal{H}}=\mathcal{E}(U \mu, \eta)=\int_{E_{0, T}} \eta \mathrm{d} \mu$ for every $\eta \in \mathcal{W}_{T} \cap C_{b}\left(E_{0, T}\right)$. Thus $\mu$ is a Revuz measure of $A$. Let $A^{\mu}:=A$. From (4.6) we easily get (4.4).

To prove the existence of $A^{\mu}$ in the general case, we choose an $\mathcal{E}$-nest $\left\{F_{n}\right\}$ of compact subsets of $E_{0, T}$ such that $\mu_{n}=\mathbf{1}_{F_{n}} \cdot \mu \in S_{0}\left(E_{0, T}\right)$. Such a nest exists by [33, Theorem 4.7]. By what has already been proved, for all $\alpha \geq 0$ and $f \in \mathcal{B}_{b}^{+}\left(E_{0, T}\right)$,

$$
E_{x} \int_{0}^{\infty} e^{-\alpha t} f\left(X_{t}\right) \mathrm{d} A_{t}^{\mu_{n}}=E_{x}^{1} \int_{0}^{\infty} e^{-\alpha t} f\left(X_{t}^{1}\right) \mathrm{d} A_{t}^{1, \bar{\mu}_{n}}
$$

for q.e. $x \in E_{0, T}$. Since the additive functional in the Revuz correspondence with a smooth measure is uniquely determined, we have $\mathrm{d} A^{\mu_{n}} \leq \mathrm{d} A^{\mu_{m}}$ for $n \leq m$ and $A_{t}^{1, \bar{\mu}_{n}}=\int_{0}^{t} \mathbf{1}_{F_{n}}\left(X_{r}\right) \mathrm{d} A_{r}^{1, \bar{\mu}}, t \geq 0, P_{x}^{1}$-a.s. We now set

$$
A_{t}^{\mu}=\sup _{n \geq 1} A_{t}^{\mu_{n}}, \quad t \geq 0 .
$$

By (4.7),

$$
\begin{aligned}
\lim _{\alpha \rightarrow \infty} \alpha E_{m_{1}} \int_{0}^{\infty} e^{-\alpha t} f\left(X_{t}\right) \mathrm{d} A_{t}^{\mu} & =\lim _{n \rightarrow \infty} \lim _{\alpha \rightarrow \infty} \alpha E_{m_{1}}^{1} \int_{0}^{\infty} e^{-\alpha t} \mathbf{1}_{F_{n}} f\left(X_{t}^{1}\right) \mathrm{d} A_{t}^{1, \bar{\mu}} \\
& =\int_{\bigcup_{n \geq 1} F_{n}} f \mathrm{~d} \bar{\mu}=\int_{E_{0, T}} f \mathrm{~d} \mu,
\end{aligned}
$$

so $A^{\mu}$ defined by (4.8) is in the Revuz correspondence with $\mu$. By [18, Lemma 1, page 182], $A^{\mu}$ is a positive natural $\mathrm{AF}$ of $\mathbb{X}$. By (2.12) and [31, Remark IV.3.6], for $m_{1}$-a.e. $x \in E_{0, T}$,

$$
\begin{aligned}
0 & =P_{x}\left(\lim _{n \rightarrow \infty} \sigma_{E_{0, T} \backslash F_{n}}<\zeta\right) \\
& =P_{x}\left(\omega \in \Omega: \lim _{n \rightarrow \infty} \inf _{t>0}\left\{X_{t}(\omega) \in E_{0, T} \backslash F_{n}\right\}<\zeta(\omega)\right) \\
& =P_{x}^{1} \circ \Pi^{-1}\left(\omega \in \Omega: \lim _{n \rightarrow \infty} \inf _{t>0}\left\{X_{t}(\omega) \in E_{0, T} \backslash F_{n}\right\}<\zeta(\omega)\right) \\
& =P_{x}^{1}\left(\omega \in \Omega^{1}: \lim _{n \rightarrow \infty} \inf _{t>0}\left\{X_{t}^{1}(\Pi(\omega)) \in E_{0, T} \backslash F_{n}\right\}<\zeta(\Pi(\omega))\right) \\
& =P_{x}^{1}\left(\omega \in \Omega^{1}: \lim _{n \rightarrow \infty} \inf _{t>0}\left\{X_{t}^{1}(\omega) \in E_{0, T} \backslash F_{n}\right\}<\zeta(\omega)\right) .
\end{aligned}
$$

Therefore, letting $n \rightarrow \infty$ in (4.7), yields (4.4). 
REMARK 4.2. Since the capacities $\mathrm{Cap}_{\psi}$ associated with $\mathcal{E}$ and the dual form $\hat{\mathcal{E}}$ coincide (see Sect. 2.1), the set of smooth measures $S\left(E_{0, T}\right)$ associated with $\mathcal{E}$ coincides with the set of smooth measures on $E_{0, T}$ associated with the dual form $\hat{\mathcal{E}}$. Therefore from Proposition 4.1 applied to $\hat{\mathcal{E}}$ and $\hat{\mathbb{X}}$ it follows that for a positive $\mu \in S\left(E_{0, T}\right)$ there exists a unique predictable $\mathrm{AF}$ of $\hat{\mathbb{X}}$ in the Revuz correspondence with $\mu$. We will denote it by $\hat{A}^{\mu}$.

In what follows, for a positive smooth measure $\mu$ on $E_{0, T}$, we put

$$
R_{\alpha} \mu(x)=E_{x} \int_{0}^{\zeta} e^{-\alpha t} \mathrm{~d} A_{t}^{\mu}, \quad \hat{R}_{\alpha} \mu(x)=\hat{E}_{x} \int_{0}^{\zeta} e^{-\alpha t} \mathrm{~d} \hat{A}_{t}^{\mu}, \quad x \in E_{0, T} .
$$

By [10, Theorem 9.3], for every $\mu \in \hat{S}_{0}\left(E_{0, T}\right)$ we have

$$
\hat{U}_{\alpha} \mu(x)=\hat{R}_{\alpha} \mu(x)
$$

for $m_{1}$-a.e. $x \in E_{0, T}$, and for every $\mu \in S_{0}\left(E_{0, T}\right)$,

$$
U_{\alpha} \mu(x)=R_{\alpha} \mu(x)
$$

for $m_{1}$-a.e. $x \in E_{0, T}$.

LEMMA 4.3. Let $\alpha \geq 0$. For any positive $\mu, v \in S\left(E_{0, T}\right)$,

$$
\int_{E_{0, T}} R_{\alpha} \nu \mathrm{d} \mu=\int_{E_{0, T}} \hat{R}_{\alpha} \mu \mathrm{d} \nu, \quad \int_{E_{0, T}} \hat{R}_{\alpha} \nu \mathrm{d} \mu=\int_{E_{0, T}} R_{\alpha} \mu \mathrm{d} \nu .
$$

Proof. First we assume that $\mu \in \hat{S}_{0}\left(E_{0, T}\right)$ and $v=\eta \cdot m_{1}$ for some $\eta \in \mathcal{B}^{+}\left(E_{0, T}\right) \cap \mathcal{H}$. Then, by (2.21), the fact that $R_{\alpha} \eta$ is an $m_{1}$-version of $G_{\alpha} \eta$ and (2.4),

$$
\int_{E_{0, T}} R_{\alpha} \eta \mathrm{d} \mu=\mathcal{E}_{\alpha}\left(R_{\alpha} \eta, \hat{U}_{\alpha} \mu\right)=\mathcal{E}_{\alpha}\left(G_{\alpha} \eta, \hat{U}_{\alpha} \mu\right)=\int_{E_{0, T}} \eta \hat{U}_{\alpha} \mu \mathrm{d} m_{1} .
$$

From this and (4.9) it follows that the first equality in (4.11) is satisfied. Now, suppose that $\mu \in S\left(E_{0, T}\right)$ and $v=\eta \cdot m_{1}$ for some $\eta \in \mathcal{B}^{+}\left(E_{0, T}\right)$. Let $\eta_{n}=\frac{n g}{1+n g}(\eta \wedge n)$ for some strictly positive $g \in \mathcal{H}$ and choose an $\mathcal{E}$-nest $\left\{F_{n}\right\}$ of compact sets such that $\mu_{n}=\mathbf{1}_{F_{n}} \cdot \mu \in \hat{S}_{0}\left(E_{0, T}\right)$. Such a nest exists by [33, Theorem 4.7]. By what has already been proved,

$$
\int_{E_{0, T}} R_{\alpha} \eta_{n} \mathrm{~d} \mu_{n}=\int_{E_{0, T}} \eta_{n} \hat{R}_{\alpha} \mu_{n} \mathrm{~d} m_{1}, \quad n \geq 1 .
$$

Letting $n \rightarrow \infty$ and using [31, Remark IV.3.6] yields the first equality in (4.11). To show it in the general case, we set $\eta_{n}=n R_{n} v$. By what has already been proved,

$$
\int_{E_{0, T}} R_{\alpha} \eta_{n} \mathrm{~d} \mu=\int_{E_{0, T}} n \hat{R}_{n} \hat{R}_{\alpha} \mu \mathrm{d} \nu, \quad n \geq 1 .
$$

By a direct calculation, we get $R_{\alpha} \eta_{n} \nearrow R_{\alpha} v$ and $n \hat{R}_{n} \hat{R}_{\alpha} \mu \nearrow \hat{R}_{\alpha} \mu$. Therefore letting $n \rightarrow \infty$ in the above equation yields the first equality in (4.11). The proof of the second equality is similar, so we omit it. 
Given a positive smooth measure $\mu$ on $E_{0, T}$, we denote by $R_{\alpha} \circ \mu$ the Borel measure on $E_{0, T}$ defined by the formula

$$
\int_{E_{0, T}} \eta d\left(R_{\alpha} \circ \mu\right)=\int_{E_{0, T}} R_{\alpha} \eta \mathrm{d} \mu, \quad \eta \in \mathcal{B}^{+}\left(E_{0, T}\right),
$$

and by $\hat{R}_{\alpha} \circ \mu$ we denote the Borel measure on $E_{0, T}$ defined by

$$
\int_{E_{0, T}} \eta d\left(\hat{R}_{\alpha} \circ \mu\right)=\int_{E_{0, T}} \hat{R}_{\alpha} \eta \mathrm{d} \mu, \quad \eta \in \mathcal{B}^{+}\left(E_{0, T}\right) .
$$

By Lemma 4.3, the measures $R_{\alpha} \circ \mu, \hat{R}_{\alpha} \circ \mu$ are absolutely continuous with respect to $m_{1}$ and

$$
R_{\alpha} \circ \mu=\left(\hat{R}_{\alpha} \mu\right) \cdot m_{1}, \quad \hat{R}_{\alpha} \circ \mu=\left(R_{\alpha} \mu\right) \cdot m_{1} .
$$

\section{Decomposition of smooth measures}

In what follows, we denote by $\langle\langle\cdot, \cdot\rangle\rangle$ the duality pairing between $\mathcal{W}_{0}^{\prime}$ and $\mathcal{W}_{0}$.

In this section, we prove that each $\mu \in \mathcal{M}_{b}\left(E_{0, T}\right)$ admits decomposition (1.7). The converse statement will be proved in Sect. 8. We start with a decomposition of elements of the space $\mathcal{W}_{0}^{\prime}$. For a proof we defer the reader to [4, Proposition 8.14] or [6, Lemma 2.24].

PROPOSITION 5.1. Let $g \in \mathcal{W}_{0}^{\prime}$. Then there exist $g_{1} \in \mathcal{V}^{\prime}, g_{2} \in \mathcal{V}$ such that

$$
\left\|g_{1}\right\| \mathcal{V}^{\prime} \leq\|g\|_{\mathcal{W}_{0}^{\prime}}, \quad\left\|g_{2}\right\| \mathcal{V} \leq\|g\|_{\mathcal{W}_{0}^{\prime}}
$$

and for every $v \in \mathcal{W}_{0}$,

$$
\langle\langle g, v\rangle\rangle=\left\langle g_{1}, v\right\rangle-\left\langle\partial_{t} v, g_{2}\right\rangle
$$

LEMMA 5.2. If $v \in \mathcal{W}_{0}$, then

$$
\alpha \hat{G}_{\alpha} v \rightarrow v \text { weakly in } \mathcal{W}_{0} \text { as } \alpha \rightarrow \infty \text {. }
$$

Proof. By [31, Proposition I.III.7], for every $w \in \mathcal{V}$,

$$
\alpha G_{\alpha} w \rightarrow w, \quad \alpha \hat{G}_{\alpha} w \rightarrow w \text { in } \mathcal{V}
$$

By (5.3), for $w \in \mathcal{V}$ we have

$$
\begin{aligned}
\left\langle\hat{\Lambda}\left(\alpha \hat{G}_{\alpha} v\right), w\right\rangle & =\mathcal{B}\left(w, \alpha \hat{G}_{\alpha} v\right)-\mathcal{E}\left(w, \alpha \hat{G}_{\alpha} v\right) \\
& =\mathcal{B}\left(w, \alpha \hat{G}_{\alpha} v\right)-\mathcal{E}\left(\alpha G_{\alpha} w, v\right) \rightarrow \mathcal{B}(w, v)-\mathcal{E}(w, v)=\langle\hat{\Lambda} v, w\rangle
\end{aligned}
$$

Since $\mathcal{V}$ is reflexive, $\left\{\hat{\Lambda}\left(\alpha \hat{G}_{\alpha} v\right)\right\}$ is weakly convergent in $\mathcal{V}^{\prime}$ as $\alpha \rightarrow \infty$. From this, (5.3) and Proposition 5.1 we get (5.2). 
REMARK 5.3. If $\mu \in S_{0}\left(E_{0, T}\right)$, then $\mu \in \mathcal{W}_{0}^{\prime}$. This follows directly from the definition of the space $S_{0}\left(E_{0, T}\right)$. Formally, the embedding $i: S_{0}\left(E_{0, T}\right) \rightarrow \mathcal{W}_{0}^{\prime}$ can be defined as follows. We first define the mapping $j: \mathcal{V} \rightarrow \mathcal{W}_{0}^{\prime}$ as

$$
\langle\langle j(u), v\rangle\rangle=\mathcal{E}_{1}(u, v), \quad u \in \mathcal{V}, v \in \mathcal{W}_{0},
$$

and then we define the embedding operator $i$ by $i(\mu)=j\left(U_{1} \mu\right)$. By the definition of $U_{1} \mu$ we then have

$$
\langle\langle i(\mu), \eta\rangle\rangle=\int_{E_{0, T}} \tilde{\eta} \mathrm{d} \mu, \quad \eta \in \mathcal{W}_{0} .
$$

PROPOSITION 5.4. Let $\mu \in \mathcal{M}_{0, b}\left(E_{0, T}\right)$. Then there exist $f \in L^{1}\left(E_{0, T} ; m_{1}\right)$ and $g \in \mathcal{W}_{0}^{\prime}$ such that

$$
\mu=f \cdot m_{1}+g
$$

i.e. for every bounded $v \in \mathcal{W}_{0}$ we have

$$
\int_{E_{0, T}} \tilde{v} d \mu=\int_{E_{0, T}} f v \mathrm{~d} m_{1}+\langle\langle g, v\rangle\rangle .
$$

Proof. Let $\mu=\mu^{+}-\mu^{-}$be the Hahn decomposition of $\mu$. Then $\mu^{+}, \mu^{-} \in \mathcal{M}_{0, b}$ $\left(E_{0, T}\right)$. Therefore, without loss of generality, we can assume that $\mu$ is positive. By [33, Theorem 4.7], there exists a nest $\left\{F_{n}\right\}$ such that $\mathbf{1}_{F_{n}} \cdot|\mu| \in S_{0}\left(E_{0, T}\right)$ for every $n \in \mathbb{N}$. Set $\mu_{n}=\mathbf{1}_{F_{n+1} \backslash F_{n}} \cdot \mu$. Then $\left|\mu_{n}\right| \in S_{0}\left(E_{0, T}\right)$ and

$$
\sum_{n=1}^{\infty} \mu_{n}=\mu
$$

because the set $\left(\bigcup_{n=1}^{\infty} F_{n}\right)^{c}$ is exceptional. Let $\mu_{n}^{\alpha}=\alpha \hat{R}_{\alpha} \circ \mu_{n}$. Then for $v \in \mathcal{W}_{0}$ we have

$$
\begin{aligned}
& \mathcal{E}_{1}\left(U_{1} \mu_{n}^{\alpha}, v\right)=\int_{E_{0, T}} \tilde{v} \mathrm{~d} \mu_{n}^{\alpha}=\int_{E_{0, T}} \alpha \hat{R}_{\alpha} v \mathrm{~d} \mu_{n}, \\
& \mathcal{E}_{1}\left(U_{1} \mu_{n}, \alpha \hat{R}_{\alpha} v\right)=\int_{E_{0, T}} \alpha \hat{R}_{\alpha} v \mathrm{~d} \mu_{n} .
\end{aligned}
$$

Hence

$$
\mathcal{E}_{1}\left(U_{1} \mu_{n}^{\alpha}, v\right)=\mathcal{E}_{1}\left(U_{1} \mu_{n}, \alpha \hat{R}_{\alpha} v\right)
$$

so by Lemma 5.2,

$$
\mathcal{E}_{1}\left(U_{1} \mu_{n}^{\alpha}, v\right) \rightarrow \mathcal{E}_{1}\left(U_{1} \mu_{n}, v\right)
$$

Let $j: \mathcal{V} \rightarrow \mathcal{W}_{0}^{\prime}$ be defined by (5.4). From (5.8) and the fact that $\mathcal{W}_{0}$ is a Hilbert space it follows that the sequence $\left\{j\left(U_{1} \mu_{n}^{\alpha}\right)\right\}$ converges to $j\left(U_{1} \mu_{n}\right)$ weakly in $\mathcal{W}_{0}^{\prime}$ as 
$\alpha \rightarrow \infty$. By the Banach-Saks theorem, there is a sequence $\left\{\alpha_{l}\right\}$ such that $\alpha_{l} \rightarrow \infty$ as $l \rightarrow \infty$ and

$$
j\left(U_{1} \hat{f}_{k}\left(\mu_{n}\right)\right) \rightarrow j\left(U_{1} \mu_{n}\right) \text { in } \mathcal{W}_{0}^{\prime}
$$

as $k \rightarrow \infty$, where

$$
\hat{f}_{k}\left(\mu_{n}\right)=\frac{1}{k} \sum_{l=1}^{k} \mu_{n}^{\alpha_{l}}
$$

Therefore one can find a subsequence $\left\{k_{n}\right\}$ such that for every $n \in \mathbb{N}$,

$$
\left\|j\left(U_{1}\left(\mu_{n}-\hat{f}_{k_{n}}\left(\mu_{n}\right)\right)\right)\right\|_{\mathcal{W}_{0}^{\prime}} \leq 2^{-n} .
$$

By (4.11), $\hat{f}_{k}\left(\mu_{n}\right)=f_{k}\left(\mu_{n}\right) \cdot m_{1}$, where

$$
f_{k}\left(\mu_{n}\right)=\frac{1}{k} \sum_{l=1}^{k} \alpha_{l} U_{\alpha_{l}} \mu_{n}
$$

Set

$$
g=\sum_{n=1}^{\infty} j\left(U_{1}\left(\mu_{n}-\left(\hat{f}_{k_{n}}\left(\mu_{n}\right)\right)\right), \quad f=\sum_{n=1}^{\infty} f_{k_{n}}\left(\mu_{n}\right)\right.
$$

By (5.9), $g \in \mathcal{W}_{0}^{\prime}$. Since $m_{1}$ is $\sigma$-finite, there exists a sequence $\left\{V_{i}\right\}$ of Borel subsets of $E_{0, T}$ such that $\bigcup_{i=1}^{\infty} V_{i}=E_{0, T}, V_{i} \subset V_{i+1}$ and $m_{1}\left(V_{i}\right)<\infty, l \in \mathbb{N}$. By (4.10) and Lemma 4.3, for every $i \in \mathbb{N}$ we have

$$
\int_{E_{0, T}} \mathbf{1}_{V_{i}} f \mathrm{~d} m_{1}=\sum_{n=1}^{\infty} \int_{E_{0, T}} \mathbf{1}_{V_{i}} f_{k_{n}}\left(\mu_{n}\right) \mathrm{d} m_{1}=\sum_{n=1}^{\infty} \int_{E_{0, T}} \frac{1}{k_{n}} \sum_{l=1}^{k_{n}} \alpha_{l} \hat{R}_{\alpha_{l}}\left(\mathbf{1}_{V_{i}}\right) \mathrm{d} \mu_{n} .
$$

Since $\alpha \hat{R}_{\alpha}$ is a Markov operator, it follows that

$$
\int_{E_{0, T}} \mathbf{1}_{V_{i}} f \mathrm{~d} m_{1} \leq \sum_{n=1}^{\infty}\left\|\mu_{n}\right\|_{T V}=\|\mu\|_{T V}
$$

Hence $\|f\|_{L^{1}\left(E_{T} ; m_{1}\right)}<\infty$ by the monotone convergence theorem. Since

$$
\int_{E_{0, T}} \tilde{v} \mathrm{~d} \mu_{n}=\mathcal{E}_{1}\left(U_{1} \mu_{n}, v\right)
$$

and

$$
\int_{E_{0, T}} f_{n_{k}}\left(\mu_{n}\right) v \mathrm{~d} m_{1}=\int_{E_{0, T}} \tilde{v} \mathrm{~d} \hat{f}_{k_{n}}\left(\mu_{n}\right)=\mathcal{E}_{1}\left(U_{1}\left(\hat{f}_{k_{n}}\left(\mu_{n}\right)\right), v\right),
$$


for every $N \in \mathbb{N}$ we have

$$
\begin{aligned}
\sum_{n=1}^{N} \int_{E_{0, T}} \tilde{v} \mathrm{~d} \mu_{n} & =\sum_{n=1}^{N} \mathcal{E}_{1}\left(U_{1}\left(\mu_{n}-\hat{f}_{k_{n}}\left(\mu_{n}\right)\right), v\right)+\sum_{n=1}^{N} \int_{E_{0, T}} f_{k_{n}}\left(\mu_{n}\right) v \mathrm{~d} m_{1} \\
& =\left\langle\left\langle\sum_{n=1}^{N} j\left(U_{1}\left(\mu_{n}-\hat{f}_{k_{n}}\left(\mu_{n}\right)\right)\right), v\right\rangle\right\rangle+\sum_{n=1}^{N} \int_{E_{0, T}}\left(f_{k_{n}}\left(\mu_{n}\right) v \mathrm{~d} m_{1} .\right.
\end{aligned}
$$

Letting $N \rightarrow \infty$ and using (5.7), (5.10) we obtain (5.6), which completes the proof of the proposition.

The idea of the proof of Proposition 5.4 is similar to that in the case of Leray-Lionstype operators on bounded domains in $\mathbb{R}^{d}$ (see [6, Theorem 2.27]). In the crucial first step we decompose $\mu$ as a sum of measures $\mu_{n} \in \mathcal{W}_{0}^{\prime}$ and then we regularize each measure $\mu_{n}$. However, contrary to [6], in the Dirichlet forms setting, we define $\mu_{n}$ by using the notion of nests (and we do not use any integral representation of smooth measures; see [6, Theorem 2.23]). Furthermore, in our general setting we cannot regularize $\mu_{n}$ by convolution. Therefore, instead of considering measures of the form $\mu_{n} * j_{1 / \alpha}$ we consider the measures $\alpha \hat{R}_{\alpha} \circ \mu_{n}$. Each measure $\alpha \hat{R}_{\alpha} \circ \mu_{n}$ has density $\alpha U_{\alpha} \mu_{n}$ with respect to the reference measure $m_{1}$ and at the same time belongs to $\mathcal{W}_{0}^{\prime}$. What is important, as in the case of regularization by convolution, our regularization by means of resolvents is a contraction with respect to the $L^{1}$-norm and with respect to the $\mathcal{W}_{0}$-norm.

REMARK 5.5. The decomposition (5.5) also holds for arbitrary $\mu \in S\left(E_{0, T}\right)$. In this case, in general, $f \notin L^{1}\left(E_{0, T} ; m_{1}\right)$ but $f$ is quasi-integrable, i.e. there exists a nest $\left\{F_{n}\right\}$ of compact subsets of $E_{0, T}$ such that $\mathbf{1}_{F_{n}} \cdot f \in L^{1}\left(E_{0, T} ; m_{1}\right), n \geq 1$. The proof of the decomposition (5.5) in case $\mu \in S\left(E_{0, T}\right)$ runs as the proof of Proposition 5.4 with the only difference that in (5.11) we replace $V_{i}$ by $F_{i}$ defined in the proof of Proposition 5.4. We then get

$$
\int_{E_{0, T}} \mathbf{1}_{F_{i}} f \mathrm{~d} m_{1} \leq\left\|\mathbf{1}_{F_{i}} \cdot \mu\right\|_{T V}, \quad i \geq 1 .
$$

Combining Proposition 5.1 with Proposition 5.4 we get the following theorem.

THEOREM 5.6. Let $\mu \in \mathcal{M}_{0, b}$. Then there exist $f \in L^{1}\left(E_{0, T} ; m_{1}\right), g_{1} \in \mathcal{V}^{\prime}$, $g_{2} \in \mathcal{V}$ such that

$$
\int_{E_{0, T}} \tilde{v} \mathrm{~d} \mu=\int_{E_{0, T}} f v \mathrm{~d} m_{1}+\left\langle g_{1}, v\right\rangle-\left\langle\partial_{t} v, g_{2}\right\rangle
$$

for every bounded $v \in \mathcal{W}_{0}$.

Note that $f, g_{1}, g_{2}$ in Theorem 5.6 are not uniquely determined. In what follows, any triplet of functions having the same properties as the triplet $\left(f, g_{1}, g_{2}\right)$ appearing in Theorem 5.6 will be called a decomposition of $\mu$.

REMARK 5.7. From (5.10) it follows that if $\mu \in \mathcal{M}_{0, b}\left(E_{0, T}\right)$ is positive, then the $L^{1}$ part $f$ of the decomposition of $\mu$ can be chosen to be positive. 


\section{Semilinear equations with right-hand side in $\mathcal{W}_{0}^{\prime}$}

In this section, using the decomposition of elements of $\mathcal{W}_{0}^{\prime}$ given in Proposition 5.1, we will show an existence and uniqueness result for the following Cauchy problem

$$
-\partial_{t} u-L_{t} u=f(\cdot, u)+g, \quad u(T, \cdot)=\varphi .
$$

In (6.1), $g \in \mathcal{W}_{0}^{\prime}, f: E_{0, T} \times \mathbb{R} \rightarrow \mathbb{R}$ and $\varphi \in L^{2}(E ; m)$.

We will need the following assumption: there exist $\lambda \in \mathbb{R}, M \geq 0$ and a positive $\varrho \in \mathcal{H}$ such for all $x \in E_{0, T}$ and $y, y^{\prime} \in \mathbb{R}$,

$$
|f(x, y)| \leq \varrho(x)+M|y|, \quad\left(f(x, y)-f\left(x, y^{\prime}\right)\right)\left(y-y^{\prime}\right) \leq \lambda\left|y-y^{\prime}\right|^{2} .
$$

DEFINITION. We say that $u \in \mathcal{V}$ is a solution of (6.1) if

$$
\left\langle\partial_{t} \eta, u\right\rangle+\mathcal{B}(u, \eta)=(\varphi, \eta(T, \cdot))_{H}+(f(\cdot, u), \eta)_{\mathcal{H}}+\langle\langle g, \eta\rangle\rangle, \quad \eta \in \mathcal{W}_{0},
$$

where $\mathcal{B}$ is defined by (2.3).

In what follows, for a function $\eta \in \mathcal{W}$ and $\varepsilon>0, t \in[0, T]$, we set $\eta^{t}=\eta \mathbf{1}_{[t, T] \times E}$ and

$$
\begin{aligned}
& \eta_{\varepsilon}^{t}(s)=0, s \in[0, t], \quad \eta_{\varepsilon}^{t}(s)=\varepsilon^{-1} \eta(t+\varepsilon)(s-t), t \in[t, t+\varepsilon], \\
& \eta_{\varepsilon}^{t}(s)=\eta(s), s \in[t+\varepsilon, T] .
\end{aligned}
$$

It is clear that for every $\eta \in \mathcal{W}, \eta_{\varepsilon}^{t} \rightarrow \eta^{t}$ strongly in $\mathcal{V}$ as $\varepsilon \searrow 0$.

THEOREM 6.1. Assume that $f$ is a Carathéodory function such that (6.2) is satisfied. Then there exists a unique solution of (6.1).

Proof. Uniqueness. Let $u_{1}, u_{2} \in \mathcal{V}$ be solutions of (6.1). Then, by (6.3),

$$
\left\langle\partial_{t} \eta, u\right\rangle+\mathcal{B}(u, \eta)=\left(f\left(\cdot, u_{1}\right)-f\left(\cdot, u_{2}\right), \eta\right)_{\mathcal{H}}, \quad \eta \in \mathcal{W}_{0},
$$

where $u=u_{1}-u_{2}$. We see that $u=G\left(f\left(\cdot, u_{1}\right)-f\left(\cdot, u_{2}\right)\right)$. In particular, $u \in \mathcal{W}_{T}$. Taking $\eta=u_{\varepsilon}^{t}$ in (6.4) and letting $\varepsilon \searrow 0$ we get

$$
\begin{aligned}
\frac{1}{2}\|u(t)\|_{H}^{2}+\int_{t}^{T} B^{(s)}(u(s), u(s)) \mathrm{d} s & =\int_{t}^{T}\left(f\left(s, u_{1}(s)\right)-f\left(s, u_{2}(s)\right), u(s)\right)_{H} \mathrm{~d} s \\
& \leq \lambda^{+} \int_{t}^{T}\|u(s)\|_{H}^{2} \mathrm{~d} s .
\end{aligned}
$$

Applying Gronwall's lemma shows that $u=0$.

Existence. Without lost of generality we may and will assume that $\lambda \leq 0$. Let $g_{1}, g_{2}$ be as in (5.1). Define $g^{\alpha} \in \mathcal{V}^{\prime}$ by

$$
\left\langle\left\langle g^{\alpha}, \eta\right\rangle\right\rangle=\left\langle\left\langle g, \alpha \hat{G}_{\alpha} \eta\right\rangle\right\rangle, \quad \eta \in \mathcal{V},
$$


and to simplify notation, let $g_{2}^{\alpha}$ stand for $\alpha \hat{G}_{\alpha} g_{2}$. Then

$$
\left\langle\left\langle g^{\alpha}, \eta\right\rangle\right\rangle=\left\langle g_{1}, \alpha \hat{G}_{\alpha} \eta\right\rangle-\left\langle\partial_{t} \eta, g_{2}^{\alpha}\right\rangle, \quad \eta \in \mathcal{V} .
$$

By [17, Theorem 6.2], there exists $u^{\alpha} \in \mathcal{W}$ such that

$\left\langle\partial_{t} \eta, u^{\alpha}\right\rangle+\mathcal{B}\left(u^{\alpha}, \eta\right)=(\varphi, \eta(T, \cdot))_{H}+\left(f\left(\cdot, u^{\alpha}\right), \eta\right)_{\mathcal{H}}+\left\langle\left\langle g^{\alpha}, \eta\right\rangle\right\rangle, \quad \eta \in \mathcal{V}$.

By this and (6.5), for every $\eta \in \mathcal{W}_{0}$ we have

$$
\begin{aligned}
& \left\langle u^{\alpha}+g_{2}^{\alpha}, \partial_{t} \eta\right\rangle+\mathcal{B}\left(u^{\alpha}+g_{2}^{\alpha}, \eta\right) \\
& \quad=(\varphi, \eta(T, \cdot))_{H}+\left(f\left(\cdot, u^{\alpha}\right), \eta\right)_{\mathcal{H}}+\left\langle g_{1}, \alpha \hat{G}_{\alpha} \eta\right\rangle+\mathcal{B}\left(g_{2}^{\alpha}, \eta\right) .
\end{aligned}
$$

Taking $\eta=\left(u^{\alpha}+g_{2}^{\alpha}\right)_{\varepsilon}^{t}$ as a test function in (6.6) and letting $\varepsilon \searrow 0$ we obtain

$$
\begin{aligned}
\frac{1}{2} \| & \left(u^{\alpha}+g_{2}^{\alpha}\right)(t) \|_{H}^{2}+\int_{t}^{T} B^{(s)}\left(\left(u^{\alpha}+g_{2}^{\alpha}\right)(s),\left(u^{\alpha}+g_{2}^{\alpha}\right)(s)\right) \mathrm{d} s \\
= & \frac{1}{2}\|\varphi\|_{H}^{2}+\int_{t}^{T}\left(f\left(\cdot, u^{\alpha}(s)\right),\left(u^{\alpha}+g_{2}^{\alpha}\right)(s)\right)_{H} \mathrm{~d} s+\left\langle g_{1}, \alpha \hat{G}_{\alpha}\left(u^{\alpha}+g_{2}^{\alpha}\right)^{t}\right\rangle \\
& +\int_{t}^{T} B^{(s)}\left(g_{2}^{\alpha}(s),\left(u^{\alpha}+g_{2}^{\alpha}\right)(s)\right) \mathrm{d} s
\end{aligned}
$$

By [31, Proposition I.3.7],

$$
\left\langle g_{1}, \alpha \hat{G}_{\alpha}\left(u^{\alpha}+g_{2}^{\alpha}\right)^{t}\right\rangle \leq C\left\|g_{1}\right\| \mathcal{V}^{\prime}\left\|u^{\alpha}+g_{2}^{\alpha}\right\| \mathcal{V} .
$$

By (6.2),

$$
\begin{aligned}
& \int_{t}^{T}\left(f\left(\cdot, u^{\alpha}(s)\right),\left(u^{\alpha}+g_{2}^{\alpha}\right)(s)\right)_{H} \mathrm{~d} s \\
& \quad \leq M \int_{t}^{T}\left\|\left(u^{\alpha}+g_{2}^{\alpha}\right)(s)\right\|_{H}^{2} \mathrm{~d} s+M\left\|g_{2}^{\alpha}\right\|_{\mathcal{H}}\left\|u^{\alpha}+g_{2}^{\alpha}\right\|_{\mathcal{H}}+\|\varrho\|_{\mathcal{H}}\left\|u^{\alpha}+g_{2}^{\alpha}\right\|_{\mathcal{H}} .
\end{aligned}
$$

Combining the above inequalities with (6.7) we get

$$
\begin{aligned}
& \frac{1}{2}\left\|\left(u^{\alpha}+g_{2}^{\alpha}\right)(t)\right\|_{H}^{2}+\int_{t}^{T} B^{(s)}\left(\left(u^{\alpha}+g_{2}^{\alpha}\right)(s),\left(u^{\alpha}+g_{2}^{\alpha}\right)(s)\right) \mathrm{d} s \\
& \leq \frac{1}{2}\|\varphi\|_{H}^{2}+M \int_{t}^{T}\left\|\left(u^{\alpha}+g_{2}^{\alpha}\right)(s)\right\|_{H}^{2} \mathrm{~d} s+M\left\|g_{2}^{\alpha}\right\| \mathcal{H}\left\|u^{\alpha}+g_{2}^{\alpha}\right\|_{\mathcal{H}} \\
& \quad+\|\varrho\|_{\mathcal{H}}\left\|u^{\alpha}+g_{2}^{\alpha}\right\| \mathcal{H}+C\left\|g_{1}\right\| \mathcal{V}^{\prime}\left\|u^{\alpha}+g_{2}^{\alpha}\right\| \mathcal{V}+\left\|g_{2}^{\alpha}\right\| \mathcal{V}\left\|u^{\alpha}+g_{2}^{\alpha}\right\| \mathcal{V}
\end{aligned}
$$

Applying Gronwall's lemma yields

$$
\begin{aligned}
& \left\|\left(u^{\alpha}+g_{2}^{\alpha}\right)(t)\right\|_{H}^{2}+\int_{0}^{T} B^{(s)}\left(\left(u^{\alpha}+g_{2}^{\alpha}\right)(s),\left(u^{\alpha}+g_{2}^{\alpha}\right)(s)\right) \mathrm{d} s \\
& \leq C(M, T)\left(\|\varphi\|_{H}^{2}+\left\|g_{2}^{\alpha}\right\| \mathcal{H}\left\|u^{\alpha}+g_{2}^{\alpha}\right\|_{\mathcal{H}}+\|\varrho\|_{\mathcal{H}}\left\|u^{\alpha}+g_{2}^{\alpha}\right\|_{\mathcal{H}}\right. \\
& \left.\quad+\left\|g_{1}\right\| \mathcal{V}^{\prime}\left\|u^{\alpha}+g_{2}^{\alpha}\right\| \mathcal{V}+\left\|g_{2}^{\alpha}\right\| \mathcal{V}\left\|u^{\alpha}+g_{2}^{\alpha}\right\| \mathcal{V}\right) .
\end{aligned}
$$


Integrating the above inequality with respect to $t$ on $[0, T]$ we get

$$
\begin{aligned}
\left\|u^{\alpha}+g_{2}^{\alpha}\right\|_{\mathcal{V}}^{2} \leq & \tilde{C}(M, T)\left(\|\varphi\|_{H}^{2}+\left\|g_{2}^{\alpha}\right\|_{\mathcal{H}}\left\|u^{\alpha}+g_{2}^{\alpha}\right\|_{\mathcal{H}}+\|\varrho\|_{\mathcal{H}}\left\|u^{\alpha}+g_{2}^{\alpha}\right\|_{\mathcal{H}}\right. \\
& \left.+\left\|g_{1}\right\| \mathcal{V}^{\prime}\left\|u^{\alpha}+g_{2}^{\alpha}\right\| \mathcal{V}+\left\|g_{2}^{\alpha}\right\| \mathcal{V}\left\|u^{\alpha}+g_{2}^{\alpha}\right\| \mathcal{V}\right)
\end{aligned}
$$

By Young's inequality,

$$
\left\|u^{\alpha}+g_{2}^{\alpha}\right\|_{\mathcal{V}}^{2} \leq \hat{C}(M, T)\left(\|\varphi\|_{H}^{2}+\left\|g_{2}^{\alpha}\right\|_{\mathcal{H}}^{2}+\|\varrho\|_{\mathcal{H}}^{2}+\left\|g_{1}\right\|_{\mathcal{V}^{\prime}}^{2}+\left\|g_{2}^{\alpha}\right\|_{\mathcal{V}}^{2}\right)
$$

By [31, Proposition I.3.7] and (5.1),

$$
\left\|u^{\alpha}+g_{2}^{\alpha}\right\|_{\mathcal{V}}^{2} \leq C^{\prime}(M, T)\left(\|\varphi\|_{H}^{2}+\|\varrho\|_{\mathcal{H}}^{2}+\|g\|_{\mathcal{W}}^{2}\right)
$$

Therefore, up to a subsequence, $\left\{u^{\alpha}+g_{2}^{\alpha}\right\}$ is weakly convergent in $\mathcal{V}$ to some $v \in \mathcal{V}$. By [31, Proposition I.3.7], $\left\{g_{2}^{\alpha}\right\}$ strongly converges in $\mathcal{V}$ to $g_{2}$, so

$$
u^{\alpha} \rightarrow u \text { weakly in } \mathcal{V}
$$

as $\alpha \rightarrow \infty$, where $u=v-g_{2}$. Observe that by (6.6) and (6.8), the sequence $\left\{u^{\alpha}+g_{2}^{\alpha}\right\}$ is bounded in $\mathcal{W}$. In particular, we have $u^{\alpha}+g_{2}^{\alpha}, u+g_{2} \in \mathcal{W}$. We now take $\eta_{\varepsilon}^{t}$ with $\eta \in \mathcal{W}_{0}$ as a test function in (6.6) and integrate by parts in the first term on the left hand side of (6.6). Next we pass to the limit with $\varepsilon \searrow 0$ and then pass to the limit with $t \nearrow T$. We then get $\left(u^{\alpha}+g_{2}^{\alpha}\right)(T)=\varphi$. Therefore (6.6) can be rewritten as

$$
-\left\langle\partial_{t}\left(u^{\alpha}+g_{2}^{\alpha}\right), \eta\right\rangle+\mathcal{B}\left(u^{\alpha}, \eta\right)=\left(f\left(\cdot, u^{\alpha}\right), \eta\right)_{\mathcal{H}}+\left\langle g_{1}, \alpha \hat{G}_{\alpha} \eta\right\rangle, \quad \eta \in \mathcal{W}_{0}
$$

It is clear that the above equation also holds true for $\eta \in \mathcal{V}$. Let $\langle\cdot, \cdot\rangle_{V^{\prime}, V}$ denote the duality between $V^{\prime}$ and $V$. Let $\eta \in \mathcal{V}$. Taking $\left(u^{\alpha}+g_{2}^{\alpha}-\eta\right)^{t}$ in place of $\eta$ in (6.10) we get

$$
\begin{aligned}
& -\int_{t}^{T}\left\langle\partial_{s}\left(u^{\alpha}+g_{2}^{\alpha}\right)(s),\left(u^{\alpha}+g_{2}^{\alpha}-\eta\right)(s)\right\rangle_{V^{\prime}, V} \mathrm{~d} s \\
& \quad+\int_{t}^{T} B^{(s)}\left(u^{\alpha}(s),\left(u^{\alpha}+g_{2}^{\alpha}-\eta\right)(s)\right) \mathrm{d} s \\
& \quad=\int_{t}^{T}\left(f\left(\cdot, u^{\alpha}\right)(s),\left(u^{\alpha}+g_{2}^{\alpha}-\eta\right)(s)\right)_{H} \mathrm{~d} s+\left\langle g_{1}, \alpha \hat{G}_{\alpha}\left(u^{\alpha}+g_{2}^{\alpha}-\eta\right)^{t}\right\rangle .
\end{aligned}
$$

By [31, Proposition I.3.7],

$$
\left\langle g_{1}, \alpha \hat{G}_{\alpha}\left(u^{\alpha}+g_{2}^{\alpha}-\eta\right)^{t}\right\rangle \rightarrow\left\langle g_{1},\left(u+g_{2}-\eta\right)^{t}\right\rangle
$$


as $\alpha \rightarrow \infty$. Thanks to the monotonicity of $L_{t}$ and $f$, we get by a pseudomonotonicity argument that

$$
\begin{aligned}
& \int_{t}^{T} B^{(s)}\left(u(s),\left(u+g_{2}-\eta\right)(s)\right) \mathrm{d} s-\int_{t}^{T}\left(f(\cdot, u)(s),\left(u+g_{2}-\eta\right)(s)\right)_{H} \mathrm{~d} s \\
& \quad \leq \liminf _{\alpha \rightarrow \infty}\left(\int_{t}^{T} B^{(s)}\left(u^{\alpha}(s),\left(u^{\alpha}+g_{2}^{\alpha}-\eta\right)(s)\right) \mathrm{d} s\right. \\
& \left.\quad-\int_{t}^{T}\left(f\left(\cdot, u^{\alpha}\right)(s),\left(u^{\alpha}+g_{2}^{\alpha}-\eta\right)(s)\right)_{H} \mathrm{~d} s\right) .
\end{aligned}
$$

Now observe that for $\eta \in \mathcal{W}_{0}$,

$$
\begin{aligned}
- & \int_{t}^{T}\left\langle\partial_{s}\left(u^{\alpha}+g_{2}^{\alpha}\right)(s),\left(u^{\alpha}+g_{2}^{\alpha}-\eta\right)(s)\right\rangle_{V^{\prime}, V} \mathrm{~d} s \\
= & -\frac{1}{2}\|\varphi-\eta(T)\|_{H}^{2}+\frac{1}{2}\left\|\left(u^{\alpha}+g_{2}^{\alpha}-\eta\right)(t)\right\|_{H}^{2} \\
& -\int_{t}^{T}\left\langle\partial_{s} \eta(s),\left(u^{\alpha}+g_{2}^{\alpha}-\eta\right)(s)\right\rangle_{V^{\prime}, V} \mathrm{~d} s .
\end{aligned}
$$

We know that $\left\{u^{\alpha}+g_{2}^{\alpha}\right\}$ is bounded in $\mathcal{W}$ and converges to $u+g_{2}$ in $\mathcal{V}$ as $\alpha \rightarrow \infty$. Therefore, by the Banach-Saks theorem, there is a sequence $\left\{\alpha_{k}\right\}$ such that $\alpha_{k} \rightarrow \infty$ as $k \rightarrow \infty$ and the Cesàro means $(1 / n) \sum_{k=1}^{n}\left(u^{\alpha_{k}}+g_{2}^{\alpha_{k}}\right)$ converge to $u+g_{2}$ strongly in $\mathcal{W}$. Since $\left(u^{\alpha}+g_{2}^{\alpha}\right)(T)=\varphi$ and $\mathcal{W} \subset C([0, T] ; H)$, it follows that $\left(u+g_{2}\right)(T)=\varphi$. This when combined with (6.14) and the proved convergences shows that for every bounded positive measurable function $\psi$ on $[0, T]$ we have

$$
\begin{aligned}
\int_{0}^{T} & \psi(t) \int_{t}^{T}\left\langle-\partial_{s}\left(u+g_{2}\right)(s),\left(u+g_{2}-\eta\right)(s)\right\rangle_{V^{\prime}, V} \mathrm{~d} s \mathrm{~d} t \\
= & \int_{0}^{T} \psi(t)\left(-\frac{1}{2}\|\varphi-\eta(T)\|_{H}^{2}+\frac{1}{2}\left\|\left(u+g_{2}-\eta\right)(t)\right\|_{H}^{2}\right. \\
& \left.-\int_{t}^{T}\left\langle\partial_{s} \eta(s),\left(u+g_{2}-\eta\right)(s)\right\rangle_{V^{\prime}, V} \mathrm{~d} s\right) \mathrm{d} t \\
\leq & \int_{0}^{T} \psi(t)\left(-\frac{1}{2}\|\varphi-\eta(T)\|_{H}^{2}\right) \mathrm{d} t+\liminf _{\alpha \rightarrow \infty} \int_{0}^{T} \psi(t)\left(\frac{1}{2}\left\|\left(u^{\alpha}+g_{2}^{\alpha}-\eta\right)(t)\right\|_{H}^{2}\right) \mathrm{d} t \\
& -\lim _{\alpha \rightarrow \infty} \int_{0}^{T} \psi(t)\left(\int_{t}^{T}\left\langle\partial_{s} \eta(s),\left(u^{\alpha}+g_{2}^{\alpha}-\eta\right)(s)\right\rangle_{V^{\prime}, V} \mathrm{~d} s\right) \mathrm{d} t \\
= & \liminf _{\alpha \rightarrow \infty} \int_{0}^{T} \psi(t) \int_{t}^{T}\left\langle-\partial_{s}\left(u^{\alpha}+g_{2}^{\alpha}\right)(s),\left(u^{\alpha}+g_{2}^{\alpha}-\eta\right)(s)\right\rangle_{V^{\prime}, V} \mathrm{~d} s \mathrm{~d} t .
\end{aligned}
$$

This and (6.11)-(6.13) show that

$$
\begin{aligned}
& \int_{0}^{T} \psi(t) \int_{t}^{T}\left\langle-\partial_{s}\left(u+g_{2}\right)(s), \eta(s)\right\rangle_{V^{\prime}, V} \mathrm{~d} s \mathrm{~d} t+\int_{0}^{T} \psi(t) \int_{t}^{T} B^{(s)}(u(s), \eta(s)) \mathrm{d} s \mathrm{~d} t \\
& \quad \leq \int_{0}^{T} \psi(t) \int_{t}^{T}(f(\cdot, u)(s), \eta(s))_{H} \mathrm{~d} s \mathrm{~d} t+\int_{0}^{T} \psi(t)\left\langle g_{1}, \eta^{t}\right\rangle_{V^{\prime}, V} \mathrm{~d} t
\end{aligned}
$$


for $\eta \in \mathcal{W}_{0}$. From this we get

$$
-\left\langle\partial_{t}\left(u+g_{2}\right), \eta\right\rangle+\mathcal{B}(u, \eta)=(f(\cdot, u), \eta)_{\mathcal{H}}+\left\langle g_{1}, \eta\right\rangle, \quad \eta \in \mathcal{W}_{0}
$$

Since we know that $\left(u+g_{2}\right)(T)=\varphi$, the above equation implies (6.3).

In the proposition below, we provide a stochastic representation of the solution of (6.1) with $f=0$ and $g \in \mathcal{W}_{0}^{\prime} \cap \mathcal{M}_{0, b}\left(E_{0, T}\right)$. It will be needed in Sect. 9.

PROPOSITION 6.2. Let $g \in \mathcal{W}_{0}^{\prime}, \varphi \in H$. Assume that there exist $\mu \in \mathcal{M}_{0, b}\left(E_{0, T}\right)$ such that

$$
\langle\langle g, \eta\rangle\rangle=\int_{E_{0, T}} \tilde{\eta} \mathrm{d} \mu
$$

for every bounded $\eta \in \mathcal{W}_{0}$. Let $u$ be a solution of (6.1) with $f=0$. Then for $m_{1}$-a.e. $x \in E_{0, T}$,

$$
u(x)=E_{x}\left(\varphi\left(X_{T}^{0}\right)+\int_{0}^{\zeta} \mathrm{d} A_{t}^{\mu}\right)
$$

Proof. We adopt the notation from the proof of Theorem 6.1. We first assume that $\varphi=0$. Let $\mu_{\alpha}=\alpha \hat{R}_{\alpha} \circ \mu$. By (4.11) and the definition of a solution of (6.1),

$$
\int_{E_{0, T}} \eta R \mu_{\alpha} \mathrm{d} m_{1}=\int_{E_{0, T}} \hat{R} \eta \mathrm{d} \mu_{\alpha}=\int_{E_{0, T}} \alpha R_{\alpha}(\hat{R} \eta) \mathrm{d} \mu=\left\langle\left\langle g^{\alpha}, \hat{R} \eta\right\rangle\right\rangle=\left(u^{\alpha}, \eta\right)_{\mathcal{H}}
$$

for every bounded $\eta \in \mathcal{W}_{0}$. By (6.9), for every $\eta \in \mathcal{H}$ we have

$$
\lim _{\alpha \rightarrow \infty}\left(u^{\alpha}, \eta\right)_{\mathcal{H}}=(u, \eta)_{\mathcal{H}}
$$

whereas by (4.11), for every $\eta \in \mathcal{W}_{0} \cap C_{b}\left(E_{0, T}\right)$,

$$
\lim _{\alpha \rightarrow \infty} \int_{E_{0, T}} \eta R \mu_{\alpha} \mathrm{d} m_{1}=\lim _{\alpha \rightarrow \infty} \int_{E_{0, T}} R \mu \cdot \alpha R_{\alpha} \eta \mathrm{d} m_{1}=\int_{E_{0, T}} \eta R \mu \mathrm{d} m_{1}
$$

Therefore $u=R \mu m_{1}$-a.e. In the general case, we put $w(x)=E_{x} \varphi\left(X_{T}^{0}\right), x \in E_{0, T}$. By [12, Theorem 3.7], $w$ is a solution of (6.1) with $f=0, g=0$. Hence $v=u-w$ is a solution of (6.1) with $f=0$ and $\varphi=0$. By what has been already proved, $v=R \mu$ $m_{1}$-a.e. Consequently, $u=w+R \mu m_{1}$-a.e.

\section{Further properties of $g_{2}$}

We know from Theorem 5.6 that each $\mu \in \mathcal{M}_{0, b}\left(E_{0, T}\right)$ admits decomposition of the form

$$
\mu=f \cdot m_{1}+g_{1}+\partial_{t} g_{2}
$$


with $f \in L^{1}\left(E_{0, T} ; m_{1}\right), g_{1} \in \mathcal{V}^{\prime}$ and $g_{2} \in \mathcal{V}$. In this section, we prove some further regularity results for $g_{2}$.

In the sequel, for a given Banach space $F$ we denote by $D([0, T] ; F)$ the set consisting of all functions $u \in L^{1}(0, T ; F)$ having a version $\tilde{u}$ (i.e. $\int_{0}^{T}\|\tilde{u}(t)-u(t)\|_{F} \mathrm{~d} t=0$ ) such that $[0, T] \ni t \mapsto \tilde{u}(t) \in F$ is càdlàg, i.e. right-continuous with left limits. Of course, $C([0, T] ; F) \subset D([0, T] ; F)$.

LEMMA 7.1. Assume that $\varphi \in L^{1}(E ; m)$ and $\rho$ is a Borel function on $E$ such that $0 \leq \rho \leq 1$ and $\int_{E} \rho \mathrm{d} m<\infty$.

(i) Let $f \in L^{1}\left(E_{0, T}\right)$. Define

$$
u(x)=E_{x}\left(\varphi\left(X_{T}^{0}\right)+\int_{0}^{\zeta} f\left(X_{t}\right) \mathrm{d} t\right), \quad x \in E_{0, T} .
$$

Then $u \in C\left([0, T] ; L^{1}(E ; \rho \cdot m)\right)$.

(ii) Let $\mu \in \mathcal{M}_{0, b}\left(E_{0, T}\right)$. Define

$$
v(x)=E_{x}\left(\varphi\left(X_{T}^{0}\right)+\int_{0}^{\zeta} \mathrm{d} A_{r}^{\mu}\right), \quad x \in E_{0, T} .
$$

Then $u \in D\left([0, T] ; L^{1}(E ; \rho \cdot m)\right)$.

Proof. Choose $\left\{\varphi_{n}\right\} \subset H \cap L^{1}(E ; m),\left\{f_{n}\right\} \subset \mathcal{H} \cap L^{1}\left(E_{0, T} ; m_{1}\right)$ and a nest $\left\{F_{n}\right\}$ so that $\left\|\varphi_{n}-\varphi\right\|_{L^{1}(E ; m)}+\left\|f_{n}-f\right\|_{L^{1}\left(E_{0, T} ; m_{1}\right)} \rightarrow 0$ as $n \rightarrow \infty$, and $\mu_{n}:=\mathbf{1}_{F_{n}} \cdot|\mu| \in$ $S_{0}\left(E_{0, T}\right)$. By Proposition 6.2, the functions

$$
\begin{aligned}
u_{n}(x)= & E_{x}\left(\varphi_{n}\left(X_{T}^{0}\right)+\int_{0}^{\zeta} f_{n}\left(X_{t}\right) \mathrm{d} t\right), \quad v_{n}(x)=E_{x}\left(\varphi_{n}\left(X_{T}^{0}\right)+\int_{0}^{\zeta} \mathrm{d} A_{r}^{\mu_{n}}\right), \\
& x \in E_{0, T},
\end{aligned}
$$

are unique solutions of the Cauchy problems

$$
-\partial_{t} u_{n}-L_{t} u_{n}=f_{n}, \quad u_{n}(T)=\varphi_{n}, \quad-\partial_{t} u_{n}-L_{t} u_{n}=\mu_{n}, \quad u_{n}(T)=\varphi_{n},
$$

respectively. Let $\left(T_{s, t}\right)_{t>s}$ (resp. $\left.\left(\hat{T}_{s, t}\right)_{t>s}\right)$ denote the semigroup determined by the form $B^{(s)}$ (resp. dual form $\hat{B}^{(s)}$ ).

It is clear that $u_{n} \in \mathcal{W} \subset C([0, T] ; H) \subset C\left([0, T] ; L^{1}(E ; \rho \cdot m)\right)$. We have

$$
\begin{aligned}
\int_{E} E_{s, x^{0}} \int_{0}^{\zeta}\left|\left(f_{n}-f\right)\left(X_{t}\right)\right| \mathrm{d} t m\left(\mathrm{~d} x^{0}\right) & \leq \int_{S}^{T}\left(\left|T_{s, t}\left(f_{n}-f\right)(t)\right|, 1\right)_{H} \mathrm{~d} t \\
& =\int_{S}^{T}\left(\left|\left(f_{n}-f\right)(t)\right|, \hat{T}_{s, t} 1\right)_{H} \mathrm{~d} t \\
& \leq\left\|f_{n}-f\right\|_{L^{1}\left(E_{0, T} ; m_{1}\right)}
\end{aligned}
$$

and

$$
\begin{aligned}
\int_{E} E_{s, x^{0}}\left|\left(\varphi_{n}-\varphi\right)\left(X_{T}^{0}\right)\right| m\left(\mathrm{~d} x^{0}\right) & =\left(T_{s, T}\left|\varphi-\varphi_{n}\right|, 1\right)_{H} \\
& =\left(\left|\varphi-\varphi_{n}\right|, \hat{T}_{s, T} 1\right)_{H} \leq\left\|\varphi-\varphi_{n}\right\|_{L^{1}(E ; m)} .
\end{aligned}
$$


Hence

$$
\sup _{t \in[0, T]}\left\|u_{n}(t)-u(t)\right\|_{L^{1}(E ; \rho \cdot m)} \leq\left\|\varphi-\varphi_{n}\right\|_{L^{1}(E ; m)}+\left\|f_{n}-f\right\|_{L^{1}\left(E_{0, T} ; m_{1}\right)} .
$$

Since $u_{n} \in C\left([0, T] ; L^{1}(E ; \rho \cdot m)\right), n \geq 1$, this proves (i).

By [12, Proposition 3.4, Theorem 3.7], $v_{n} \in D([0, T] ; H) \subset D\left([0, T] ; L^{1}(E ; \rho\right.$. $m)$ ). Let $v=\delta_{\{s\}} \otimes m$. Then, by Lemma 4.3

$$
\begin{aligned}
\int_{E}\left|E_{s, x^{0}} \int_{0}^{\zeta} \mathrm{d} A_{t}^{\mu}-E_{s, x^{0}} \int_{0}^{\zeta} \mathrm{d} A_{t}^{\mu_{n}}\right| m\left(\mathrm{~d} x^{0}\right) & \leq E_{v} \int_{0}^{\zeta} \mathrm{d} A_{t}^{\left|\mu-\mu_{n}\right|} \\
& =\int_{E_{0, T}} \hat{R} \nu d\left|\mu_{n}-\mu\right| .
\end{aligned}
$$

Since $v \in S\left(E_{0, T}\right)$, by using Lemma 4.3 with $\alpha=0$ we get

$$
\|\hat{R} v\|_{\infty}=\sup _{\|\eta\|_{L^{1}} \leq 1} \int_{E_{0, T}} \eta \hat{R} v \mathrm{~d} m_{1}=\sup _{\|\eta\|_{L^{1}} \leq 1} \int_{E_{0, T}} R \eta \mathrm{d} v=\sup _{\|\eta\|_{L^{1}} \leq 1}\|(R \eta)(s)\|_{L^{1}(E ; m)} .
$$

But $\int_{E} R \eta\left(s, x^{0}\right) m\left(\mathrm{~d} x^{0}\right) \leq \int_{s}^{T}\left(T_{s, t} \eta(t), 1\right)_{H} \mathrm{~d} t \leq 1$. Hence $\|\hat{R} \nu\|_{\infty} \leq 1$. Therefore from (7.2) and (7.3) it follows that

$$
\begin{aligned}
\sup _{t \in[0, T]}\left\|v_{n}(t)-v(t)\right\|_{L^{1}(E ; \rho \cdot m)} & \leq\left\|\varphi-\varphi_{n}\right\|_{L^{1}(E ; m)}+\left\|\mu_{n}-\mu\right\|_{T V} \\
& =\left\|\varphi-\varphi_{n}\right\|_{L^{1}(E ; m)}+|\mu|\left(E_{0, T} \backslash F_{n}\right) .
\end{aligned}
$$

Since $v_{n} \in D\left([0, T] ; L^{1}(E ; \rho \cdot m)\right), n \geq 1$, and the right-hand side of the above inequality converges to zero as $n \rightarrow \infty$, we get (ii).

The following corollary extends [6, Lemma 2.29].

COROLLARY 7.2. Let $\mu \in \mathcal{M}_{0, b}\left(E_{0, T}\right)$ and $\left(f, g_{1}, g_{2}\right),\left(\bar{f}, \bar{g}_{1}, \bar{g}_{2}\right)$ be two decompositions of $\mu$. Then $g_{2}-\bar{g}_{2} \in C\left([0, T] ; L^{1}(E ; \rho \cdot m)\right)$ for any function $\rho$ on $E$ such that $0 \leq \rho \leq 1$ and $\int_{E} \rho \mathrm{d} m=1$.

Proof. For every bounded $\eta \in \mathcal{W}_{0}$, we have

$$
\left\langle\partial_{t} \eta, g_{2}-\bar{g}_{2}\right\rangle=-\left\langle g_{1}-\bar{g}_{1}, \eta\right\rangle-\int_{E_{0, T}}(f-\bar{f}) \eta \mathrm{d} m_{1} .
$$

Hence, for every bounded $\eta \in \mathcal{W}_{0}$,

$$
\left\langle\partial_{t} \eta, g_{2}-\bar{g}_{2}\right\rangle+\mathcal{B}\left(g_{2}-\bar{g}_{2}, \eta\right)=-\left\langle g_{1}-\bar{g}_{1}, \eta\right\rangle+\langle\chi, \eta\rangle-\int_{E_{0, T}}(f-\bar{f}) \eta \mathrm{d} m_{1},
$$

where $\chi$ is an element of $\mathcal{V}^{\prime}$ such that $\langle\chi, \eta\rangle=\mathcal{B}\left(g_{2}-\bar{g}_{2}, \eta\right), \eta \in \mathcal{V}$. Let $u=g_{2}-\bar{g}_{2}$ and $v \in \mathcal{W}_{T}$ be a solution to the Cauchy problem

$$
-\partial_{t} v-L_{t} v=-\left(g_{1}-\bar{g}_{1}\right)+\chi, \quad v(T)=0 .
$$


Then

$$
\left\langle\partial_{t} \eta, u-v\right\rangle+\mathcal{B}(u-v, \eta)=-\int_{E_{0, T}}(f-\bar{f}) \eta \mathrm{d} m_{1}
$$

for all bounded $\eta \in \mathcal{W}_{0}$. By Proposition 6.2, for $m_{1}$-a.e. $x \in E_{0, T}$ we have

$$
(u-v)(x)=E_{x} \int_{0}^{\zeta}(\bar{f}-f)\left(X_{t}\right) \mathrm{d} t .
$$

By Lemma 7.1, $u-v \in C\left([0, T] ; L^{1}(E ; \rho \cdot m)\right)$. Since $v \in \mathcal{W}_{T} \subset C([0, T] ; H) \subset$ $C\left([0, T] ; L^{1}(E ; \rho \cdot m)\right)$, we get the desired result.

PROPOSITION 7.3. Let $\mu \in \mathcal{M}_{0, b}\left(E_{0, T}\right)$ and let $\left(f, g_{1}, g_{2}\right)$ be a decomposition of $\mu$. Let $\rho$ be a Borel function on $E$ such that $0 \leq \rho \leq 1$ and $\int_{E} \rho \mathrm{d} m<\infty$.

(i) $g_{2} \in D\left([0, T] ; L^{1}(E ; \rho \cdot m)\right)$ and $g_{2}(T-)=0$.

(ii) For $t \in(0, T)$, let $\mu_{t}$ be the measure defined as $\mu_{t}(B)=\mu(\{t\} \times B), B \in \mathcal{B}(E)$.

Then

$$
\mu_{t}=\left(g_{2}(t-)-g_{2}(t)\right) \cdot m \text {. }
$$

Proof. Without loss of generality we may assume that $f=0$ (otherwise, we consider the measure $v=\mu-f \cdot m_{1}$ instead of $\mu$ ). Then $\mu \in \mathcal{W}_{0}^{\prime}$. By Proposition 6.2, the function $u(x)=E_{x} \int_{0}^{\zeta} \mathrm{d} A_{r}^{\mu}, x \in E$, is a solution to the problem

$$
-\partial_{t} u-L_{t} u=\mu, \quad u(T, \cdot)=0,
$$

so by the definition,

$$
\left\langle\partial_{t} \eta, u\right\rangle+\mathcal{B}(u, \eta)=\left\langle g_{1}, \eta\right\rangle-\left\langle\partial_{t} \eta, g_{2}\right\rangle, \quad \eta \in \mathcal{W}_{0} .
$$

Hence

$$
\left\langle\partial_{t} \eta, u-g_{2}\right\rangle+\mathcal{B}\left(u-g_{2}, \eta\right)=\chi(\eta), \quad \eta \in \mathcal{W}_{0},
$$

where $\chi(\eta)=-\mathcal{B}\left(g_{2}, \eta\right)+\left\langle\eta, g_{1}\right\rangle$. From this we conclude that $u-g_{2} \in \mathcal{W}_{T} \subset$ $C([0, T] ; H) \subset D\left([0, T] ; L^{1}(E ; \rho \cdot m)\right)$. By Lemma 7.1,u $\in D\left([0, T] ; L^{1}(E ; \rho \cdot m)\right)$. Therefore $g_{2} \in D\left([0, T] ; L^{1}(E ; \rho \cdot m)\right)$. For $t \in[0, T)$, we set

$$
\begin{aligned}
& \rho_{\varepsilon}^{t}(s)=0, s \in[0, t-\varepsilon], \quad \rho_{\varepsilon}^{t}(s)=\varepsilon^{-1}(s-t+\varepsilon), s \in[t-\varepsilon, t] \\
& \rho_{\varepsilon}^{t}(s)=1, s \in[t, T] .
\end{aligned}
$$

Let $\xi \in V \cap C_{c}(E)$ and $\eta_{\varepsilon}^{t}=\rho_{\varepsilon}^{t} \xi$. Because $u \in D\left([0, T] ; L^{1}(E ; \rho \cdot m)\right)$ and $u-g_{2} \in C([0, T] ; H)$, replacing $\eta$ by $\eta_{\varepsilon}^{t}$ in (7.5) and (7.6), and then letting $\varepsilon \downarrow 0$ and $t \uparrow T$ shows that $u(T-)=0$ and $\left(u-g_{2}\right)(T)=0$. Hence $g_{2}(T-)=0$. Taking $\eta_{\varepsilon}^{t}$ as a test function in (5.12) and letting $\varepsilon \downarrow 0$ we obtain

$$
\int_{[t, T) \times E} \xi \mathrm{d} \mu=\left\langle\mathbf{1}_{[t, T) \times E} g_{1}, \xi\right\rangle-\int_{E} \xi g_{2}(t-) \mathrm{d} m
$$

from which we conclude that (7.4) is satisfied. 
The following proposition is a counterpart to [25, Theorem 1.1].

PROPOSITION 7.4. Let $\mu \in \mathcal{M}_{0, b}\left(E_{0, T}\right)$. Then for every $\varepsilon>0$ there exists $a$ measure $\mu_{\varepsilon} \in S_{0}\left(E_{0, T}\right)-S_{0}\left(E_{0, T}\right)$ such that $\left\|\mu_{\varepsilon}-\mu\right\|_{T V} \leq \varepsilon$ and $\mu_{\varepsilon}$ admits decomposition of the form (5.12) with $f=0$ and $g_{2} \in \mathcal{V} \cap L^{\infty}\left(E_{0, T} ; m_{1}\right)$.

Proof. Without loss of generality we can assume that $\mu$ is positive. Let $\left\{F_{n}^{1}\right\}$ be a nest such that $\mathbf{1}_{F_{n}^{1}} \cdot \mu \in S_{0}\left(E_{0, T}\right)$. Let $u=R \mu, F_{n}^{2}=\{u \leq n\}$ and $F_{n}=F_{n}^{1} \cap F_{n}^{2}$, $\mu_{n}=\mathbf{1}_{F_{n}} \cdot \mu$ and $u_{n}=R \mu_{n}$. By Itô's formula (see also 9.4),

$\left|u_{n}(x)\right|^{2} \leq 2 E_{x} \int_{0}^{\zeta} u_{n}\left(X_{t-}\right) \mathbf{1}_{F_{n}}\left(X_{t-}\right) \mathrm{d} A_{t}^{\mu} \leq 2 n E_{x} \int_{0}^{\zeta} \mathbf{1}_{F_{n}}\left(X_{t-}\right) \mathrm{d} A_{t}^{\mu}=2 n u_{n}(x)$.

Hence $u_{n}(x) \leq 2 n$ for q.e. $x \in E_{0, T}$. Consequently, $u_{n} \in L^{\infty}\left(E_{0, T} ; m_{1}\right)$. By Proposition $6.2, u_{n}$ is a solution to the Cauchy problem

$$
-\partial_{t} u_{n}-L_{t} u_{n}=\mu_{n}, \quad u_{n}(T)=0 .
$$

In other words, for every bounded $v \in \mathcal{W}_{0}$,

$$
\int_{E_{0, T}} \tilde{v} \mathrm{~d} \mu_{n}=\left\langle\chi_{n}, v\right\rangle+\left\langle\partial_{t} v, u_{n}\right\rangle,
$$

where $\chi_{n}$ is an element of $\mathcal{V}^{\prime}$ such that $\left\langle\chi_{n}, \eta\right\rangle=\mathcal{B}\left(u_{n}, v\right), v \in \mathcal{V}$. Thus $\mu_{n}=$ $\chi_{n}+\partial_{t} u_{n}$, i.e. $\mu_{n}$ admits the decomposition (5.12) with $f=0$ and $g_{2}:=u_{n} \in$ $\mathcal{V} \cap L^{\infty}\left(E_{0, T} ; m_{1}\right)$. Furthermore, $\left\|\mu_{n}-\mu\right\|_{T V}=\mu\left(E_{0, T} \backslash F_{n}\right) \rightarrow 0$ as $n \rightarrow \infty$, which completes the proof.

It is known (see [25, Example 3.1]) that not every bounded smooth measure can be written in the form (7.1) with bounded $g_{2}$. We are going to show that $g_{2}$ is always quasi-bounded with respect to the capacity $c_{2}$. To this end, we first recall the definition of a parabolic potential (see, e.g., [26]).

DEFINITION. A measurable function $u \in \mathcal{V} \cap L^{\infty}(0, T ; H)$ is called a parabolic potential if for every positive $v \in \mathcal{W}_{0}$,

$$
\left\langle\partial_{t} v, u\right\rangle+\mathcal{B}(u, v) \geq 0 .
$$

The set of parabolic potentials will be denoted by $\mathcal{P}^{2}$. By [27, Proposition I.1], for every $u \in \mathcal{P}^{2}$ there exists a unique Borel measure $\mu_{u}$ such that for every $\eta \in$ $\mathcal{W}_{0} \cap C_{b}\left(E_{0, T}\right)$

$$
\left\langle\partial \eta_{t}, u\right\rangle+\mathcal{B}(u, \eta)=\int_{E_{0, T}} \eta \mathrm{d} \mu_{u}
$$

Let $\mu \in S_{0}$ be positive. Then, by [13, Proposition 3.1], $\mu \in \mathcal{P}^{2}$ and $\mu_{R \mu}=\mu$. Following [28], for $u \in \mathcal{P}^{2}$ we set

$$
\|u\|_{\Lambda}^{2}=\operatorname{ess}_{\sup }(0, T)\|u(t)\|_{H}^{2}+\|u\|_{\mathcal{V}}^{2} .
$$


For an open set $U \subset E_{0, T}$, we define

$$
c_{1}(U)=\inf \left\{\|\eta\|_{\Lambda}: \eta \in \mathcal{P}^{2}, \eta \geq \mathbf{1}_{U} m_{1} \text {-a.e. }\right\} .
$$

By [28, Theorem 1], there exists $\alpha>0$ such that

$$
c_{0} \leq \alpha c_{1}
$$

For $k \geq 0$, we set

$$
T_{k}(s)=\max \{-k, \min \{k, s\}\}, \quad s \in \mathbb{R} .
$$

LEMMA 7.5. Let $\mu \in \mathcal{M}_{0, b}^{+}$. Then $T_{k}(R \mu) \in \mathcal{P}^{2}, k \geq 0$, and there exists $c>0$ such that

$$
\left\|T_{k}(R \mu)\right\|_{\Lambda}^{2} \leq c k\|\mu\|_{T V}
$$

Proof. By [12, Theorem 3.12], $T_{k}(R \mu) \in \mathcal{V}$. Let $\left\{F_{n}\right\}$ be a $\mathrm{Cap}_{\psi}$-nest of compact sets such that $\mu_{n}:=\mathbf{1}_{F_{n}} \cdot \mu \in S_{0}$. Since $\left\{F_{n}\right\}$ is a Cap $\psi$-nest, $R \mu_{n} \nearrow R \mu \mathrm{Cap}_{\psi}$-q.e., hence $m_{1}$-a.e. Since $R \mu_{n} \in \mathcal{P}^{2}$, by [27, Corollary I.1], $T_{k}\left(R \mu_{n}\right) \in \mathcal{P}^{2}$. Hence, by [26, Lemma III-1], there exists $c>0$ such that

$$
\left\|T_{k}\left(R \mu_{n}\right)\right\|_{\Lambda}^{2} \leq c k\left\|\mu_{T_{k}\left(R \mu_{n}\right)}\right\|_{T V}
$$

By [26, Lemma II-5],

$$
\left\|T_{k}\left(R \mu_{n}\right)\right\|_{\Lambda}^{2} \leq c k\left\|\mu_{T_{k}\left(R \mu_{n}\right)}\right\|_{T V} \leq c k\left\|\mu_{R \mu_{n}}\right\|_{T V}=c k\left\|\mu_{n}\right\|_{T V} \leq c k\|\mu\|_{T V} .
$$

By [12, Theorem 3.12], $\sup _{n \geq 1}\left\|T_{k}\left(R \mu_{n}\right)\right\| \mathcal{V}<\infty$. Since $T_{k}\left(R \mu_{n}\right) \rightarrow T_{k}(R \mu) m_{1^{-}}$ a.e. as $n \rightarrow \infty$, it follows that, up to a subsequence, $T_{k}\left(R \mu_{n}\right) \rightarrow T_{k}(R \mu)$ weakly in $\mathcal{V}$. It is clear that $T_{k}(R \mu)$ satisfies (7.8), which when combined with (7.10) implies that $T_{k}(R \mu) \in \mathcal{P}^{2}$ and the desired inequality holds true.

PROPOSITION 7.6. Assume that $\mu \in \mathcal{M}_{b}\left(E_{0, T}\right)$ is of the form (1.7). Then $g_{2}$ has an $m_{1}$-version $\tilde{g}_{2}$ which is $c_{0}$-quasi-bounded.

Proof. Set $v=\mu-f \cdot m_{1}$ and

$$
v(x)=-E_{x} \int_{0}^{\zeta} \mathrm{d} A_{t}^{v},
$$

where $A^{v}$ is a natural $\mathrm{AF}$ of $\mathbb{X}$ in the Revuz correspondence with $v$. By Proposition $6.2, v$ is a solution to (6.1) with $f=0, \varphi=0$ and $g$ replaced by $-v$. Observe that $w=v-g_{2}$ is a solution to the Cauchy problem

$$
-\partial_{t} w-L_{t} w=-g_{1}-\chi, \quad w(T)=0
$$


where $\chi$ is an element of $\mathcal{V}^{\prime}$ such that $\langle\chi, \eta\rangle=\mathcal{B}\left(g_{2}, \eta\right), \eta \in \mathcal{V}$. Since $g_{1}+\chi \in \mathcal{V}^{\prime}$, we have $w \in \mathcal{W}_{T}$. Therefore

$$
g_{2}=w-E . A_{\zeta}^{v} \quad m_{1} \text {-a.e. }
$$

for some $w \in \mathcal{W}_{T}$. By [27, Theorem III.1], $w$ has an $m_{1}$-version $\tilde{w}$ which is $c_{0}$-quasicontinuous, so $c_{0}$-quasi-bounded. Therefore we only need to show that $v_{+}(x):=$ $E_{x} A_{\zeta}^{\nu^{+}}$and $v_{-}(x):=E_{x} A_{\zeta}^{v^{-}}$have $m_{1}$-versions which are $c_{0}$-quasi-bounded. We will show this for $v_{+}$. The proof for $v_{-}$is analogous. Let $v_{+}^{k}=T_{k}\left(v_{+}\right)$. By Lemma 7.5, $v_{+}^{k} \in \mathcal{P}^{2}$, so by [27, Corollary III.3], there exists an $m_{1}$-version $\tilde{v}_{+}^{k}$ of $v_{+}^{k}$ which is $c_{0}$-quasi-l.s.c. Hence $\tilde{v}_{+}:=\sup _{k \geq 1} \tilde{v}_{+}^{k}$ is also $c_{0}$-quasi-1.s.c., and of course, it is an $m_{1}$-version of $v_{+}$. By Lemma 7.5 and (7.9),

$$
c_{0}\left(\tilde{v}_{+}>n\right)=c_{0}\left(\tilde{v}_{+}^{n+1}>n\right) \leq \alpha n^{-2}\left\|\left(v_{+}^{n+1}\right)\right\|_{\Lambda}^{2} \leq 2 c \alpha n^{-1}\left\|v^{+}\right\|_{T V} .
$$

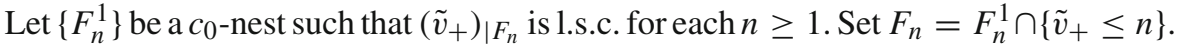
Then $F_{n}$ is closed. Moreover,

$$
c_{0}\left(E_{0, T} \backslash F_{n}\right) \leq c_{0}\left(\tilde{v}_{+}>n\right)+c_{0}\left(E_{0, T} \backslash F_{n}^{1}\right) \rightarrow 0
$$

as $n \rightarrow \infty$, which proves that $\tilde{v}_{+}$is $c_{0}$-quasi-bounded.

\section{Smoothness of measures in $\mathcal{W}_{0}^{\prime}$}

We already know that each bounded smooth measure admits a decomposition of the form (1.7). The problem whether a bounded measure admitting decomposition (1.7) is smooth is more delicate. In this section, we give positive answer to this question.

In the proof of our result, we will make use of Fukushima's decomposition, which we now recall. For an AF $A$ of $\mathbb{X}$ its energy is defined by

$$
e(A)=\frac{1}{2} \lim _{\alpha \rightarrow \infty} \alpha^{2} E_{m_{1}} \int_{0}^{\infty} e^{-\alpha t}\left|A_{t}\right|^{2} \mathrm{~d} t
$$

whenever the limit exists in [0, $\infty$ ]. By [31, Proposition IV.1.8], each $u \in \mathcal{W}$ has a quasi-continuous $m_{1}$-modification, which we will denote by $\tilde{u}$. By [32, Theorem 4.5], for any $w \in \mathcal{W}$ there exists a unique martingale $\mathrm{AF}$ of $\mathbb{X}$ of finite energy $M^{[u]}$ and a unique continuous AF of $\mathbb{X}$ of zero energy $N^{[u]}$ such that

$$
\tilde{u}\left(X_{t}\right)-\tilde{u}\left(X_{0}\right)=M_{t}^{[u]}+N_{t}^{[u]}, \quad t \geq 0, \quad P_{x} \text {-a.s. }
$$

for q.e. $x \in E_{0, T}$. The decomposition (8.1) is called Fukushima's decomposition.

PROPOSITION 8.1. Assume that $u \in \mathcal{W}_{T}$. Then

$$
e\left(M^{[u]}\right) \leq \mathcal{B}(u, u) .
$$


Proof. Let $A_{t}^{[u]}=\tilde{u}\left(X_{t}\right)-\tilde{u}\left(X_{0}\right), t \geq 0$. We have

$$
\begin{aligned}
\alpha^{2} E_{m_{1}} \int_{0}^{\infty} e^{-\alpha t}\left|A_{t}^{[u]}\right|^{2} \mathrm{~d} t & =\alpha^{2} \int_{E_{0, T}}\left(R_{\alpha} u-2 u R_{\alpha} u+\alpha^{-1} u\right) \mathrm{d} m_{1} \\
& =2 \alpha\left(u-\alpha R_{\alpha} u, u\right)_{\mathcal{H}}-\alpha\left(u^{2}, 1-\alpha \hat{R}_{\alpha} 1\right)_{\mathcal{H}} \\
& =2 \mathcal{E}\left(\alpha G_{\alpha} u, u\right)-\alpha\left(u^{2}, 1-\alpha \hat{R}_{\alpha} 1\right)_{\mathcal{H}} .
\end{aligned}
$$

By (2.15), $\alpha \hat{R}_{\alpha} 1(x)=1-\hat{E}_{x} e^{-\alpha \zeta}, x \in E_{0, T}$. Hence

$$
\alpha\left(u^{2}, 1-\alpha \hat{R}_{\alpha} 1\right)_{\mathcal{H}}=\int_{E_{0, T}} \alpha|u|^{2} \hat{E}_{x} e^{-\alpha \zeta} m_{1}(\mathrm{~d} x) \geq \int_{0}^{T} \alpha|u(s)|_{H}^{2} e^{-\alpha s} \mathrm{~d} s,
$$

which converges to $\|u(0)\|_{H}^{2}$ as $\alpha \rightarrow \infty$. By the above calculations,

$$
e\left(A^{[u]}\right)=\limsup _{\alpha \rightarrow \infty}\left(\mathcal{E}\left(\alpha G_{\alpha} u, u\right)-\frac{1}{2} \alpha\left(u^{2}, 1-\alpha \hat{R}_{\alpha} 1\right)_{\mathcal{H}}\right),
$$

so by [30, Proposition 2.7],

$$
e\left(A^{[u]}\right) \leq \mathcal{E}(u, u)-\frac{1}{2}\|u(0)\|_{H}^{2}=\mathcal{B}(u, u) .
$$

On the other hand, by (8.1), $e\left(A^{[u]}\right)=e\left(M^{[u]}\right)$, which proves the proposition.

LEMMA 8.2. Assume that $A, A^{n}, n \geq 1$, are additive functionals of $\mathbb{X}$.

(i) If $E_{\eta \cdot m_{1}} \sup _{t \geq 0}\left|A_{t}\right|^{p}<\infty$ for some $p \geq 1$ and strictly positive $\eta \in \mathcal{B}\left(E_{0, T}\right)$, then $E_{x} \sup _{t \geq 0}\left|A_{t}\right|^{p}<\infty$ for q.e. $x \in E_{0, T}$.

(ii) If $E_{\eta \cdot m_{1}} \sup _{t \geq 0}\left|A_{t}^{n}-A_{t}^{m}\right|^{p} \rightarrow 0$ as $n, m \rightarrow \infty$ for some $p \geq 0$ and strictly positive $\eta \in \mathcal{B}\left(E_{0, T}\right)$, then there exists a subsequence (still denoted by $n$ ) such that $E_{x} \sup _{t \geq 0}\left|A_{t}^{n}-A_{t}^{m}\right|^{p} \rightarrow 0$ as $n, m \rightarrow \infty$ for q.e. $x \in E_{0, T}$.

Proof. We will prove (ii). The proof of (i) is analogous. We may assume that

$$
E_{\eta \cdot m_{1}} \sup _{t \geq 0}\left|A_{t}^{n}-A_{t}^{n+1}\right|^{p} \leq 2^{-n}, \quad n \geq 1,
$$

and $\int_{E_{0, T}} \eta \mathrm{d} m_{1}=1$. Let $\tilde{m}_{1}=\eta \cdot m_{1}$ and

$$
B=\liminf _{n \rightarrow \infty}\left\{\sup _{t \geq 0}\left|A_{t}^{n}-A_{t}^{n+1}\right|^{p}>2^{-2 n}\right\}, \quad N=\left\{x \in E_{0, T}: P_{x}(B)>0\right\} .
$$

It is clear that $N$ is a nearly Borel set. We will show that $\operatorname{Cap}_{\psi}(N)=0$. Since $\operatorname{Cap}_{\psi}$ is a Choquet capacity, we may and will assume that $N$ is compact. We have

$$
P_{\tilde{m}_{1}}\left(\sigma_{N}<\infty\right) \leq P_{\tilde{m}_{1}}\left(X_{\sigma_{N}} \in N\right)=P_{\tilde{m}_{1}}\left(E_{X_{\sigma_{N}}} \mathbf{1}_{B}>0\right) .
$$

By the strong Markov property,

$$
P_{\tilde{m}_{1}}\left(E_{X_{\sigma_{N}}} \mathbf{1}_{B}>0\right)=P_{\tilde{m}_{1}}\left(E_{x}\left(\mathbf{1}_{B} \circ \theta_{\sigma_{N}} \mid \mathcal{F}_{\sigma_{N}}\right)>0\right) .
$$


Since $A$ is additive,

$$
\begin{aligned}
B \circ \theta_{\sigma_{N}} & =\liminf _{n \rightarrow \infty}\left\{\sup _{t \geq 0}\left|A_{t}^{n} \circ \theta_{\sigma_{N}}-A_{t}^{n+1} \circ \theta_{\sigma_{N}}\right|^{p}>2^{-n}\right\} \\
& \subset \liminf _{n \rightarrow \infty}\left\{\sup _{t \geq 0}\left|A_{t}^{n}-A_{t}^{n+1}\right|^{p}>2^{-n+p}\right\}=: B^{\prime} .
\end{aligned}
$$

Hence

$$
P_{\tilde{m}_{1}}\left(\sigma_{N}<\infty\right) \leq \int_{E_{0, T}} P_{x}\left(E_{x}\left(\mathbf{1}_{B^{\prime}} \mid \mathcal{F}_{\sigma_{N}}\right)>0\right) \tilde{m}_{1}(\mathrm{~d} x)
$$

But by (8.2) and the Borel-Cantelli lemma, $P_{\tilde{m}_{1}}\left(B^{\prime}\right)=0$, which implies that $P_{x}\left(B^{\prime}\right)=$ 0 for $m_{1}$-a.e. $x \in E_{0, T}$. Hence, for $m_{1}$-a.e. $x \in E_{0, T}, P_{x}\left(E_{x}\left(\mathbf{1}_{B^{\prime}} \mid \mathcal{F}_{\sigma_{N}}\right)>0\right)=0$, so $P_{\tilde{m}_{1}}\left(\sigma_{N}<\infty\right)=0$. Equivalently, $\operatorname{Cap}_{\psi}(N)=0$, as claimed.

LEMMA 8.3. The set $C=\left\{\sum_{i=1}^{n} \xi_{i} v_{i}: \xi_{i} \in C_{c}^{\infty}((0, T)), v_{i} \in V \cap C_{c}(E)\right\}$ is dense in $\mathcal{W}_{0}^{2}$.

Proof. Follows from [22, Lemma 1.1].

THEOREM 8.4. Let $\mu \in \mathcal{M}_{b}\left(E_{0, T}\right)$. If there exist $f \in L^{1}\left(E_{0, T} ; m_{1}\right), g_{1} \in \mathcal{V}^{\prime}$, $g_{2} \in \mathcal{V}$ such that (5.12) is satisfied for all $\eta \in \mathcal{W}_{0} \cap C_{b}\left(E_{0, T}\right)$, then $\mu \in \mathcal{M}_{0, b}\left(E_{0, T}\right)$.

Proof. Since the measure $f \cdot m_{1}$ is smooth, without loss of generality we may assume that $f=0$. Let $\Phi$ be a functional on $\mathcal{W}_{0}$ defined by the right-hand side of (5.12) (with $f=0$ ). It is clear that $\Phi \in \mathcal{W}_{0}^{\prime}$. Let $u \in \mathcal{V}$ be a solution to (6.1) with $\varphi=0, f=0$ and $g=\Phi$.

Step 1. We will show that $u$ is a difference of excessive functions. By (5.12) and the definition of solution to (6.1), for every $\eta \in \mathcal{W}_{0} \cap C_{b}\left(E_{0, T}\right)$,

$$
|\mathcal{E}(u, \eta)| \leq\|\mu\|_{T V}\|\eta\|_{\infty}
$$

Let $\mathcal{C}=\left\{\sum_{i=1}^{n} \xi_{i} v: \xi_{i} \in H^{1}(0, T), \xi_{i}(0)=0, v \in V \cap C_{c}(E)\right\}$. Then $\mathcal{C} \subset$ $\mathcal{W}_{0} \cap C_{b}\left(E_{0, T}\right)$, so (8.3) holds for every $\eta \in \mathcal{C}$. By Lemma 8.3, $\mathcal{C}$ is dense in $\mathcal{W}_{0}^{2}$. Let $\eta \in \mathcal{W}_{0}^{2}$ be bounded. Write $c=\|\eta\|_{\infty}$ and choose $\left\{\eta_{n}\right\} \subset \mathcal{C}$ such that and $\eta_{n} \rightarrow \eta$ in $\mathcal{W}_{0}^{2}$. Then $T_{c}\left(\eta_{n}\right) \rightarrow T_{c}(\eta)=\eta$ in $\mathcal{W}_{0}^{2}$, so (8.3) holds for every bounded $\eta \in \mathcal{W}_{0}^{2}$. Suppose now that $\eta \in \mathcal{W}_{0}$ and $\eta$ is bounded. Then $\alpha \hat{R}_{\alpha} \eta \in \mathcal{W}_{0}^{2},\left\|\alpha \hat{R}_{\alpha} \eta\right\|_{\infty} \leq\|\eta\|_{\infty}$ and $\alpha \hat{R}_{\alpha} \eta \rightarrow \eta$ in $\mathcal{W}_{0}$ as $\alpha \rightarrow \infty$. Therefore (8.3) holds for every bounded $\eta \in \mathcal{W}_{0}$. By [1, Proposition 4.5], there exist excessive functions $v$ and $w$ such that $u=v-w$ $m_{1}$-a.e. and for every $\eta \in \mathcal{H} \cap L^{\infty}\left(E_{0, T} ; m_{1}\right)$,

$$
\begin{gathered}
\int_{E_{0, T}}(v+w)|\eta| \mathrm{d} m_{1}<\infty, \\
\frac{1}{t} \int_{E_{0, T}}\left|P_{t} v-v\right| \eta \mathrm{d} m_{1}+\frac{1}{t} \int_{E_{0, T}}\left|P_{t} w-w\right| \eta \mathrm{d} m_{1} \leq c\|\eta\|_{\infty} .
\end{gathered}
$$


Since each excessive function finite $m_{1}$-a.e. is finite q.e., we may assume that $v(x)+$ $w(x)<\infty, x \in E_{0, T} \backslash N$, where $N$ is some $m_{1}$-inessential set (see [10, Proposition $6.12])$.

Step 2. Let

$$
\tilde{u}=v-w, \quad u_{n}=n R_{n} \tilde{u}, \quad v_{n}=\left(n \tilde{u}-n^{2} R_{n} \tilde{u}\right) \cdot m_{1} .
$$

By [31, Proposition I.3.7], $u_{n} \rightarrow u$ strongly in $\mathcal{V}$. By Fukushima's decomposition, there exists an $m_{1}$-inessential set $N$ such that for every $x \in E_{0, T} \backslash N$,

$$
u_{n}\left(X_{t}\right)=u_{n}\left(X_{0}\right)-\int_{0}^{t} \mathrm{~d} A_{r}^{v_{n}}+\int_{0}^{t} d M_{r}^{\left[u_{n}\right]}, \quad t \leq \zeta, \quad P_{x} \text {-a.s. }
$$

We will show the uniform convergence of $\left\{M^{\left[u_{n}\right]}\right\}$ as $n \rightarrow \infty$. Let $\left\langle M^{\left[u_{n}\right]}\right\rangle$ denote the sharp bracket of $M^{\left[u_{n}\right]}$ (see, e.g, [8, Sect. A.3]). We have

$$
\begin{aligned}
e\left(M^{\left[u_{n}\right]}\right) & =\frac{1}{2} \lim _{\alpha \rightarrow \infty} \alpha^{2} E_{m_{1}} \int_{0}^{\infty} e^{-\alpha t} E_{m_{1}}\left\langle M^{\left[u_{n}\right]}\right\rangle_{t} \mathrm{~d} t \\
& =\frac{1}{2} \lim _{\alpha \rightarrow \infty} \int_{0}^{\infty} s e^{-s} \frac{\alpha}{s} E_{m_{1}}\left\langle M^{\left[u_{n}\right]}\right\rangle_{s / \alpha} \mathrm{d} s .
\end{aligned}
$$

Since $t \mapsto E_{m_{1}}\left\langle M^{\left[u_{n}\right]}\right\rangle_{t}$ is subadditive, $(1 / t) E_{m_{1}}\left\langle M^{\left[u_{n}\right]}\right\rangle_{t}$ increases as $t$ decreases, and $\lim _{t \downarrow 0}(1 / t) E_{m_{1}}\left\langle M^{\left[u_{n}\right]}\right\rangle_{t}=\sup _{t>0}(1 / t) E_{m}\left\langle M^{\left[u_{n}\right]}\right\rangle_{t}$. Therefore, letting $\alpha \rightarrow \infty$ in (8.7), shows that

$$
e\left(M^{\left[u_{n}\right]}\right)=\frac{1}{2} \sup _{t>0} \frac{1}{t} E_{m_{1}}\left\langle M^{\left[u_{n}\right]}\right\rangle_{t} \cdot \int_{0}^{\infty} s e^{-s} \mathrm{~d} s=\frac{1}{2} \sup _{t>0} \frac{1}{t} E_{m_{1}}\left\langle M^{\left[u_{n}\right]}\right\rangle_{t} .
$$

Hence

$$
E_{m_{1}}\left\langle M^{\left[u_{n}\right]}\right\rangle_{\zeta} \leq 2 T e\left(M^{\left[u_{n}\right]}\right)
$$

so applying Doob's inequality we get

$$
E_{m_{1}} \sup _{t \geq 0}\left|M_{t}^{\left[u_{n}\right]}-M_{t}^{\left[u_{m}\right]}\right|^{2} \leq 8 T e\left(M^{\left[u_{n}-u_{m}\right]}\right)
$$

for all $n, m \geq 1$. By Proposition 8.1 and Lemma 8.2, there exists an $m_{1}$-inessential set $N$ such that, up to a subsequence,

$$
\lim _{n, m \rightarrow \infty} E_{x} \sup _{t \geq 0}\left|M_{t}^{\left[u_{n}\right]}-M_{t}^{\left[u_{m}\right]}\right|^{2}=0, \quad x \in E_{0, T} \backslash N .
$$

Step 3. We will show that there exists a natural $\mathrm{AF} A$ of $\mathbb{X}$ of finite variation and a martingale $\mathrm{AF} M$ of $\mathbb{X}$ such that

$$
\tilde{u}\left(X_{t}\right)=\tilde{u}\left(X_{0}\right)-\int_{0}^{t} \mathrm{~d} A_{r}+\int_{0}^{t} \mathrm{~d} M_{r}, \quad t \leq \zeta, \quad P_{x} \text {-a.s. }
$$


for $x \in E_{0, T} \backslash N$. Set

$$
M_{t}=\liminf _{n \rightarrow \infty} M_{t}^{\left[u_{n}\right]}, \quad A_{t}=\liminf _{n \rightarrow \infty} A_{t}^{v_{n}}, \quad t \geq 0 .
$$

By the definition of $\tilde{u}$, for every $x \in E_{0, T}$,

$$
u_{n}\left(X_{t}\right) \rightarrow \tilde{u}\left(X_{t}\right), \quad t \geq 0, \quad P_{x} \text {-a.s. }
$$

Therefore, letting $n \rightarrow \infty$ in (8.6), shows that (8.8) is satisfied. Moreover, $M$ is a square integrable martingale under the measure $P_{x}$ for $x \in E_{0, T} \backslash N$, and

$$
E_{x} \sup _{t \geq 0}\left|M_{t}^{\left[u_{n}\right]}-M_{t}\right|^{2} \rightarrow 0, \quad A_{t}=\lim _{n \rightarrow \infty} A_{t}^{v_{n}}, \quad t \geq 0, P_{x} \text {-a.s. }
$$

for every $x \in E_{0, T} \backslash N$. By [2, Theorem III.5.7], $v(X), w(X)$ are càdlàg supermartingales under the measure $P_{x}$ for $x \in E_{0, T} \backslash N$. In particular, $\tilde{u}(X)$ and $A$ are càdlàg processes under $P_{x}$ for $x \in E_{0, T} \backslash N$. By the resolvent identity,

$$
\begin{aligned}
R\left|v_{n}\right| & =n R\left|u-n R_{n} u\right| \leq n R\left|v-n R_{n} v\right|+n R\left|w-n R_{n} w\right| \\
& =n R\left(v-n R_{n} v\right)+n R\left(w-n R_{n} w\right)=n R_{n} v+n R_{n} w \leq v+w .
\end{aligned}
$$

Hence

$$
\sup _{n \geq 1} E_{x}\left|A^{v_{n}}\right|_{\zeta} \leq v(x)+w(x)<\infty, \quad x \in E_{0, T} \backslash N .
$$

Let $t_{i}^{k}=i T / 2^{k}, i=0, \ldots, 2^{k}$. Since $A$ is càdlàg,

$$
|A|_{T}=\lim _{k \rightarrow \infty} \sum_{i=1}^{k}\left|A_{t_{i}^{k}}-A_{t_{i-1}^{k}}\right|=\sup _{k \geq 1} \sum_{i=1}^{k}\left|A_{t_{i}^{k}}-A_{t_{i-1}^{k}}\right| .
$$

Hence

$$
\begin{aligned}
E_{x}|A|_{T} & =E_{x} \lim _{k \rightarrow \infty} \sum_{i=1}^{k}\left|A_{t_{i}^{k}}-A_{t_{i-1}^{k}}\right|=\lim _{k \rightarrow \infty} E_{x} \sum_{i=1}^{k}\left|A_{t_{i}^{k}}-A_{t_{i-1}^{k}}\right| \\
& \leq \lim _{k \rightarrow \infty} \liminf _{n \rightarrow \infty} E_{x} \sum_{i=1}^{k}\left|A_{t_{i}^{k}}^{v_{n}}-A_{t_{i-1}^{k}}^{v_{n}}\right| \leq \liminf _{n \rightarrow \infty} E_{x} \sup _{k \geq 1} \sum_{i=1}^{k}\left|A_{t_{i}^{k}}^{v_{n}}-A_{t_{i-1}^{k}}^{v_{n}}\right| \\
& =\liminf _{n \rightarrow \infty} E_{x} \lim _{k \rightarrow \infty} \sum_{i=1}^{k}\left|A_{t_{i}^{k}}^{v_{n}}-A_{t_{i-1}^{k}}^{v_{n}}\right|=\liminf _{n \rightarrow \infty} E_{x}\left|A^{v_{n}}\right|_{T} .
\end{aligned}
$$

From this and (8.11) we get

$$
E_{x}|A|_{\zeta}<\infty, \quad x \in E_{0, T} \backslash N .
$$

Let

$\Lambda=\left\{\omega \in \Omega: A_{t}(\omega)=\limsup _{n \rightarrow \infty} A_{t}^{n}(\omega), t \geq 0 A .(\omega)\right.$ is càdlàg of finite variation $\}$. 
By what has already been proved, $P_{x}(\Lambda)=1$ for $x \in E_{0, T} \backslash N$. Observe that $\theta_{t}(\Lambda) \subset$ $\Lambda$. Moreover, for all $s, t \geq 0$ and $\omega \in \Lambda$,

$$
A_{t}\left(\theta_{s}(\omega)\right)=\lim _{n \rightarrow \infty} A_{t}^{v_{n}}\left(\theta_{s}(\omega)\right)=\lim _{n \rightarrow \infty}\left(A_{t+s}^{v_{n}}(\omega)-A_{s}^{v_{n}}(\omega)\right)=A_{t+s}(\omega)-A_{s}(\omega) .
$$

Of course, the same relation holds for $A^{+}, A^{-}$. Thus $A^{+}, A^{-}$are positive natural AFs of $\mathbb{X}$ with the defining set $\Lambda$ and exceptional set $N$. This implies that $M$ is a martingale AF of $\mathbb{X}$.

Step 4. Let $v^{+}$(resp. $v^{-}$) be the Revuz measure associated with $A^{+}$(resp. $A^{-}$) (see [10, Sect. 8]). To complete the proof it suffices to show that $v \in \mathcal{M}_{0, b}\left(E_{0, T}\right)$ and $v=\mu$. Since $M$ is a uniformly integrable martingale,

$$
\tilde{u}(x)=E_{x} A_{\zeta}, \quad x \in E_{0, T} \backslash N .
$$

We have $\tilde{u}=u_{+}-u_{-}$, where

$$
u_{+}(x):=E_{x} A_{\zeta}^{+}, \quad u_{-}(x):=E_{x} A_{\zeta}^{-}, \quad x \in E_{0, T} \backslash N .
$$

It is clear that $u_{+}, u_{-}$are natural potentials (see [2, Definition IV.4.17] and the comments following the definition). By the construction of functions $v, w$ (see [1, Proposition 4.2]),

$$
v(x) \leq u_{+}(x), \quad w(x) \leq u_{-}(x), \quad x \in E_{0, T} \backslash N .
$$

Therefore $v, w$ are natural potentials, and by [2, Theorem IV.4.22], $v=E . A_{\zeta}^{1}, w=$ $E . A_{\zeta}^{2}$ for some positive natural AFs $A^{1}, A^{2}$ of $\mathbb{X}$. By the minimality argument,

$$
v(x)=u_{+}(x), \quad w(x)=u_{-}(x), \quad x \in E_{0, T} \backslash N .
$$

From this, (8.5), (8.10) and [10, Theorem 9.3] it follows that for every positive $\eta \in$ $C_{b}\left(E_{0, T}\right)$,

$$
\frac{1}{t} E_{\eta \cdot m}|A|_{t} \leq \frac{1}{t} \int_{E_{0, T}}\left|P_{t} v-v\right| \eta \mathrm{d} m_{1}+\frac{1}{t} \int_{E_{0, T}}\left|P_{t} w-w\right| \eta \mathrm{d} m_{1} \leq c\|\eta\|_{\infty} .
$$

By [10, Theorem 8.7], for every positive $\eta \in C_{b}\left(E_{0, T}\right)$,

$$
\lim _{t \downarrow 0} \frac{1}{t} E_{\eta \cdot m}|A|_{t}=\int_{E_{0, T}} \eta \mathrm{d}|v| .
$$

Therefore $\int_{E_{0, T}} \eta \mathrm{d}|\nu|<\infty$ for any positive $\eta \in C_{b}\left(E_{0, T}\right)$. Consequently, $v \in$ $\mathcal{M}_{b}\left(E_{0, T}\right)$. By the very definition of $v^{+}$and $v^{-}$, if $\operatorname{Cap}_{\psi}(B)=0$, then $|v|(B)=0$, so $v \in \mathcal{M}_{0, b}\left(E_{0, T}\right)$. By [10, Theorem 9.3], $\tilde{u}=R v$ q.e. By the definition of a solution to (6.1) and (5.12),

$$
\mathcal{E}(u, \eta)=\int_{E_{0, T}} \eta \mathrm{d} \mu
$$


for $\eta \in \mathcal{W}_{0} \cap C_{b}\left(E_{0, T}\right)$. On the other hand, for every $\eta \in \mathcal{W}_{0} \cap C_{b}\left(E_{0, T}\right)$,

$$
\begin{aligned}
\mathcal{E}(u, \eta) & =\lim _{t \downarrow 0} \frac{1}{t}\left(u, \eta-\hat{T}_{t} \eta\right)=\lim _{t \downarrow 0} \frac{1}{t}\left(u-T_{t} u, \eta\right) \\
& =\lim _{t \downarrow 0} \frac{1}{t} E_{\eta \cdot m_{1}} \int_{0}^{t} \mathrm{~d} A_{r}^{v}=\int_{E_{0, T}} \eta \mathrm{d} \nu .
\end{aligned}
$$

Hence $\mu=v$.

\section{Decomposition of measures and additive functionals}

In this section, we study the structure of the additive functional $A^{\mu}$ in the Revuz correspondence with $\mu \in \mathcal{M}_{0, b}\left(E_{0, T}\right)$. Specifically, we want to get deeper understanding of the nature of jumps of $A^{\mu}$ and their relation to the decomposition $\left(f, g_{1}, g_{2}\right)$ of $\mu$ given in Theorem 5.6. Before proceeding, remarks concerning two special cases of $\mu$ are in order.

If $\mu=f \cdot m_{1}$, then

$$
A_{t}^{\mu}=\int_{0}^{t} f\left(X_{r}\right) \mathrm{d} r, \quad t \geq 0 .
$$

If $\mu=g_{1}$, then

$$
A_{t}^{\mu}=-N_{t}^{[u]}, \quad t \geq 0,
$$

where $u \in \mathcal{W}_{T}$ is a unique solution to the Cauchy problem

$$
\left(\partial_{t}+L_{t}\right) u=-g_{1}, \quad u(T)=0,
$$

and $N^{[u]}$ is the continuous AF from Fukushima's decomposition (8.1). Indeed, by Proposition 6.2, $u=R \mu m_{1}$-a.e. In other words, for q.e. $x \in E_{0, T}$ we have

$$
\tilde{u}(x)=E_{x} A_{\zeta}^{\mu}
$$

Let $M_{t}^{x}=E_{x}\left(A_{\zeta}^{\mu} \mid \mathcal{F}_{t}\right)-\tilde{u}\left(X_{0}\right), t \geq 0$. Because of standard perfection procedure (see, e.g., [8, Lemma A.3.6]), there is a martingale AF $M$ of $\mathbb{X}$ such that $M_{t}=M_{t}^{x}, t \geq 0$, $P_{x}$-a.s. for q.e. $x \in E_{0, T}$. From (9.3) and the strong Markov property we obtain

$$
\tilde{u}\left(X_{t}\right)-\tilde{u}\left(X_{0}\right)=-A_{t}^{\mu}+M_{t}, \quad t \geq 0
$$

(see [14, Remark 3.3] for more details). Since $\tilde{u}$ is quasi-continuous and the filtration is quasi-left continuous, from (9.4) it follows that $A^{\mu}$ is continuous. It is clear that $M$ is a martingale $\mathrm{AF}$ of $\mathbb{X}$. Let $u_{n}=n R_{n} u$. Then

$$
u_{n}\left(X_{t}\right)-u_{n}\left(X_{0}\right)=N_{t}^{\left[u_{n}\right]}+M_{t}^{\left[u_{n}\right]}, \quad t \geq 0,
$$


and by Itô's formula,

$$
u_{n}\left(X_{t}\right)-u_{n}\left(X_{0}\right)=-A_{t}^{\mu_{n}}+M_{t}^{n}, \quad t \geq 0,
$$

where $\mu_{n}=n\left(u_{n}-u\right) \cdot m_{1}$ and $M_{t}^{n}=E_{x}\left(A_{\zeta}^{\mu_{n}} \mid \mathcal{F}_{t}\right)-\tilde{u}_{n}\left(X_{0}\right) P_{x}$-a.s. for q.e. $x \in E_{0, T}$. By an elementary calculation (see, e.g., [8, page 245]), $A^{\mu_{n}}$ is of zero energy. By uniqueness of Fukushima's decomposition, $-A^{\mu_{n}}=N^{\left[u_{n}\right]}$. We know that $u_{n} \rightarrow \tilde{u}$ q.e. and $u_{n} \rightarrow u$ in $\mathcal{V}$, so by Proposition 8.1, $N^{\left[u_{n}\right]} \rightarrow N^{[u]}$. Also, by [11, Theorem 3.3], $A_{t}^{\mu_{n}} \rightarrow A_{t}^{\mu}, t \geq 0$. Thus $-A^{\mu}=N^{[u]}$.

We see that in both special cases considered above the additive functionals corresponding to $\mu$ are continuous. This suggests that the jumps of $A^{\mu}$ stem from the component $g_{2}$ of the decomposition of $\mu$. In what follows we will show that this is indeed true and we will make this statement more precise.

Following [12] we adopt the following definition.

DEFINITION. We say that a Borel measurable function $u$ on $E_{0, T}$ is quasi-càdlàg if for q.e. $x \in E_{0, T}$ the process $t \mapsto u\left(X_{t}\right)$ is càdlàg on $[0, T-\tau(0))$ under the measure $P_{x}$.

Since $A^{\mu}$ is predictable, by [5, Chapter IV, Theorem $\left.88 \mathrm{~B}\right]$, there is a sequence $\left\{\tau_{n}\right\}$ of predictable stopping times exhausting the jumps of $A^{\mu}$, i.e.

$$
\left\{\Delta A^{\mu} \neq 0\right\}=\bigcup_{n=1}^{\infty}\left[\left[\tau_{n}\right]\right], \quad\left[\left[\tau_{n}\right]\right] \cap\left[\left[\tau_{m}\right]\right]=\emptyset, \quad n \neq m,
$$

where $\left[\left[\tau_{n}\right]\right]$ denotes the graph of $\tau_{n}$.

In what follows, we denote by $A^{\mu, c}$ the continuous part of $A^{\mu}$ and by $A^{\mu, d}$ the pure jump part of $A^{\mu}$. For a given càdlàg process $Y$, we write $\Delta Y_{t}=Y_{t}-Y_{t-}$, where $Y_{t-}=\lim _{s \nearrow t} Y_{s}$.

THEOREM 9.1. Let $\mu \in \mathcal{M}_{0, b}\left(E_{0, T}\right)$ and $\left(f, g_{1}, g_{2}\right)$ be a decomposition of $\mu$ from Theorem 5.6. Then

(i) $g_{2}$ has a quasi-càdlàg $m_{1}$-version $\tilde{g}_{2}$.

(ii) Let $Y=\tilde{g}_{2}(X)$. For q.e. $x \in E_{0, T}$ we have

$$
A_{t}^{\mu, d}=\sum_{\tau_{n} \leq t} \Delta Y_{\tau_{n}} \quad P_{x} \text {-a.s. }
$$

Proof. Set $v=\mu-f \cdot m_{1}$ and

$$
v(x)=-E_{x} \int_{0}^{\zeta} \mathrm{d} A_{t}^{v}, \quad x \in E_{0, T},
$$

where $A^{v}$ is a natural $\mathrm{AF}$ of $\mathbb{X}$ in the Revuz correspondence with $v$. By Proposition $6.2, v$ is quasi-càdlàg and it is a solution to (6.1) with $f=0, \varphi=0$ and $g$ replaced by $-v$. By (7.11), $g_{2}=v-w m_{1}$-a.e. for some $w \in \mathcal{W}_{T}$. Write

$$
\tilde{g}_{2}=v-\tilde{w}
$$


By the argument used to prove (9.4), there is a martingale AF $M^{v}$ of $\mathbb{X}$ such that

$$
v\left(X_{t}\right)-v\left(X_{0}\right)=M_{t}^{v}+A_{t}^{v}, \quad t \geq 0, \quad P_{x} \text {-a.s. }
$$

for q.e. $x \in E_{0, T}$. By Fukushima's decomposition (8.1),

$$
\widetilde{w}\left(X_{t}\right)-\widetilde{w}\left(X_{0}\right)=M_{t}^{[w]}+N_{t}^{[w]}, \quad t \geq 0, \quad P_{x} \text {-a.s. }
$$

for q.e. $x \in E_{0, T}$. Hence

$$
\tilde{g}_{2}\left(X_{t}\right)-\tilde{g}_{2}\left(X_{0}\right)=M_{t}^{v}-M_{t}^{[w]}+A_{t}^{v}-N_{t}^{[w]}, \quad t \geq 0, \quad P_{x} \text {-a.s. }
$$

for q.e. $x \in E_{0, T}$. Since the filtration $\left(\mathcal{F}_{t}\right)$ is quasi-left continuous, by [8, Theorems A.3.2, A.3.6], the martingale AFs $M^{v}$ and $M^{[w]}$ admit no predictable jump. Therefore from (9.9) and continuity of the functionals $N^{[w]}$ and $A^{f \cdot m_{1}}$ we get the result.

REMARK 9.2. (i) By (9.8), $\tilde{g}_{2}$ is quasi-continuous if and only if $v$ is quasicontinuous, which in turn is equivalent to the continuity of $A^{\mu}$.

(ii) By [10, Theorem 16.8], there is a positive Borel function $h: E_{0, T} \rightarrow \mathbb{R}$ such that

$$
\Delta A_{t}^{\mu, d}=h\left(X_{t-}\right), \quad t \geq 0, \quad P_{x} \text {-a.s. }
$$

for q.e. $x \in E_{0, T}$. By this and (9.7),

$$
\tilde{g}_{2}\left(X_{\tau_{n}}\right)-\tilde{g}_{2}(X)_{\tau_{n}-}=h\left(X_{\tau_{n}-}\right)=h\left(X_{\tau_{n}}\right) \quad P_{x} \text {-a.s. }
$$

the second equality being a consequence of the quasi-continuity of the filtration $\left(\mathcal{F}_{t}\right)$. We see that if $\tilde{g}_{2}$ is not quasi-continuous, then the jumps of $\tilde{g}_{2}(X)$ which are not produced by $X$ are produced by $\tilde{g}_{2}$. The size of these jumps is described by $h$.

EXAMPLE 9.3. Assume that $B^{(t)}=B^{(0)}$ for $t \in[0, T]$ and there exists a strictly positive $\beta: E \rightarrow \mathbb{R}$ such that $\beta \in L^{1}(E ; m) \cap C(E)$ (for instance, we may take $E$ to be a bounded open subset $D$ of $\mathbb{R}^{d}$ and consider the classical Dirichlet form on $L^{2}\left(D ; \mathrm{d} x^{0}\right)$ defined as $\left.B^{(t)}(u, v)=\int_{D} \nabla u\left(x^{0}\right) \nabla v\left(x^{0}\right) \mathrm{d} x^{0}, u, v \in H_{0}^{1}(D)\right)$. Let $a \in(0, T)$. We define $\mu$ on $E_{0, T}$ by

$$
\mu\left(\mathrm{d} t \mathrm{~d} x^{0}\right)=\left(\delta_{\{a\}}+\ell\right)(\mathrm{d} t) \otimes \beta\left(x^{0}\right) m\left(\mathrm{~d} x^{0}\right),
$$

where $\ell$ is the Lebesgue measure on $[0, T]$. Clearly $\mu \in \mathcal{M}_{b}\left(E_{0, T}\right)$. Moreover, if $\operatorname{Cap}_{\psi}(B)=0$ for some Borel set $B \subset E_{0, T}$, then $m_{1}(B)=0$ and Cap $\psi\left(\{t\} \times B_{t}\right)=0$ for every $t \in(0, T]$, where $B_{t}=\left\{x^{0} \in E:\left(t, x^{0}\right) \in B\right\}$. From this, [22, (4.4)] and $[23,(6.2 .24)]$ it follows that $m\left(B_{t}\right)$ for $t \in(0, T]$. In particular, $m\left(B_{a}\right)=0$, and consequently $\mu(B)=0$. Thus $\mu \in \mathcal{M}_{0, b}\left(E_{0, T}\right)$. Let

$$
\alpha(t)=\mathbf{1}_{[a, T]}(t)+t, \quad g_{2}\left(t, x^{0}\right)=\alpha(t) \beta\left(x^{0}\right), \quad\left(t, x^{0}\right) \in E_{0, T} .
$$

Then

$$
\mu=\partial_{t} g_{2}
$$


By (2.6), $g_{2}\left(X_{t}\right)=\alpha(v(t)) \beta\left(X_{v(t)}^{0}\right), t \geq 0, P_{x}$-a.s. for q.e. $x \in E_{0, T}$, from which it follows that $g_{2}$ is quasi-càdlàg. Furthermore, under the measure $P_{x}$ with $x=\left(s, x^{0}\right)$, for every predictable $\tau$ we have

$$
\Delta g_{2}(X)_{\tau}=(\alpha(s+\tau)-\alpha((s+\tau)-)) \beta\left(X_{s+\tau}^{0}\right)= \begin{cases}0, & s+\tau \neq a \\ \beta\left(X_{a}^{0}\right), & s+\tau=a\end{cases}
$$

Consequently, $g_{2}$ is not quasi-continuous. Finally, we note that

$$
\Delta A_{t}^{\mu, d}=\mathbf{1}_{\{a\}}(s+t) \beta\left(X_{s+t}^{0}\right)=h\left(X_{t}\right), \quad t \in[0, T-s], \quad P_{s, x^{0} \text {-a.s. },}
$$

where $h\left(t, x^{0}\right)=\mathbf{1}_{\{a\}}(t) \beta\left(x^{0}\right),\left(t, x^{0}\right) \in E_{0, T}$.

\section{Acknowledgements}

This work was supported by Polish National Science Centre (Grant No. 2016/23/B/ST1 /01543).

Open Access. This article is distributed under the terms of the Creative Commons Attribution 4.0 International License (http://creativecommons.org/licenses/by/4.0/), which permits unrestricted use, distribution, and reproduction in any medium, provided you give appropriate credit to the original author(s) and the source, provide a link to the Creative Commons license, and indicate if changes were made.

Publisher's Note Springer Nature remains neutral with regard to jurisdictional claims in published maps and institutional affiliations.

\section{REFERENCES}

[1] Beznea, L., Cîmpean, I.: Quasimartingales associated to Markov processes. Trans. Amer. Math. Soc. 370 (2018) 7761-7787.

[2] Blumenthal, R.M., Getoor, R.K.: Markov Processes and Potential Theory. Academic Press, New York and London 1968.

[3] Boccardo, L., Gallouët, T., Orsina, L.: Existence and uniqueness of entropy solutions for nonlinear elliptic equations with measure data. Ann. Inst. H. Poincaré Anal. Non Linéaire 13 (1996) 539-551.

[4] Brezis, H.: Functional analysis, Sobolev spaces and partial differential equations. Springer, New York, 2011.

[5] Dellacherie, C., Meyer, P.-A.: Probabilities and Potential. North-Holland Publishing Co., Amsterdam, 1978.

[6] Droniou, J., Porretta, A., Prignet, A.: Parabolic Capacity and Soft Measures for Nonlinear Equations. Potential Anal. 19 (2003) 99-161.

[7] Fukushima, M.: On semi-martingale characterizations of functionals of symmetric Markov processes. Electron. J. Probab. 4 (1999), no. 18, 32 pp.

[8] Fukushima, M., Oshima, Y., Takeda, M.: Dirichlet Forms and Symmetric Markov Processes. Second revised and extended edition. Walter de Gruyter, Berlin 2011.

[9] Fukushima, M., Sato, K., Taniguchi, S.: On the closable parts of pre-Dirichlet forms and the fine supports of underlying measures. Osaka J. Math. 28 (1991) 517-535.

[10] Getoor R.K., Sharpe, M.J.: Naturality, standardness, and weak duality for Markov processes. Z. Wahrsch. Verw. Gebiete 67 (1984) 1-62.

[11] Klimsiak, T.: Reflected BSDEs on filtered probability spaces. Stochastic Process. Appl. 125 (2015) 4204-4241. 
[12] Klimsiak, T.: Semi-Dirichlet forms, Feynman-Kac functionals and the Cauchy problem for semilinear parabolic equations. J. Funct. Anal. 268 (2015) 1205-1240.

[13] Klimsiak, T.: Obstacle problem for evolution equations involving measure data and operator corresponding to semi-Dirichlet form. J. Evol. Equ. 18 (2018) 681-713.

[14] Klimsiak, T., Rozkosz, A.: Renormalized solutions of semilinear equations involving measure data and operator corresponding to Dirichlet form. NoDEA Nonlinear Differential Equations Appl. 22 (2015) 1911-1934.

[15] Klimsiak, T., Rozkosz, A.: On the structure of bounded smooth measures associated with a quasiregular Dirichlet form. Bull. Polish Acad. Sci. Math. 65 (2017) 45-56.

[16] Klimsiak, T., Rozkosz, A.: On the structure of diffuse measures for parabolic capacities. arXiv: 1808.06422.

[17] Lions, J.-L.: Quelques Méthodes de Résolutions des Problèmes aux Limites Non Linéaires. Dunod, Gauthier Villars, Paris 1969.

[18] Meyer, P.-A.: Fonctionnelles multiplicatives et additives de Markov. Ann. Inst. Fourier, 12 (1962) $125-230$.

[19] Ma, Z.-M., Röckner, M.: Introduction to the Theory of (Non-Symmetric) Dirichlet Forms. SpringerVerlag, Berlin 1992.

[20] Oshima, Y.: On a construction of Markov processes associated with time dependent Dirichlet spaces. Forum Math. 4 (1992) 395-415.

[21] Oshima, Y.: Some properties of Markov processes associated with time dependent Dirichlet forms. Osaka J. Math. 29 (1992) 103-127.

[22] Oshima, Y.: Time-dependent Dirichlet forms and related stochastic calculus. Infin. Dimens. Anal. Quantum Probab. Relat. Top. 7 (2004) 281-316.

[23] Oshima, Y.: Semi-Dirichlet Forms and Markov Processes. Walter de Gruyter, Berlin 2013.

[24] Petitta, F.: Renormalized solutions of nonlinear parabolic equations with general measure data. Ann. Mat. Pura Appl. 187 (2008) 563-604.

[25] Petitta, F., Ponce, A.C., Porretta, A.: Diffuse measures and nonlinear parabolic equations. J. Evol. Equ. 11 (2011) 861-905.

[26] Pierre, M.: Problèmes d'évolution avec contraintes unilatérales et potentiel paraboliques. Comm. Partial Differential Equations 4 (1979) 1149-1197.

[27] Pierre, M.: Représentant précis d'un potentiel parabolique. Séminaire de Théorie du Potentiel, Paris, No. 5, Lecture Notes in Math. 814 (1980) 186-228.

[28] Pierre, M.: Parabolic capacity and Sobolev spaces. SIAM J. Math. Anal. 14 (1983) 522-533.

[29] Revuz, D.: Mesures associees aux fonctionnelles additives de Markov I. Trans. Amer. Math. Soc. 148 (1970) 501-531.

[30] Stannat, W.: Dirichlet forms and Markov processes: a generalized framework including both elliptic and parabolic cases. Potential Anal. 8 (1998) 21-60.

[31] Stannat, W.: The Theory of Generalized Dirichlet Forms and Its Applications in Analysis and Stochastics. Mem. Amer. Math. Soc. 142 (1999), no. 678.

[32] Trutnau, G.: Stochastic calculus of generalized Dirichlet forms and applications to stochastic differential equations in infinite dimensions. Osaka J. Math. 37 (2000) 315-343.

[33] Trutnau, G.: Analytic properties of smooth measures in the non-sectorial case, (Stochastic Analysis of Jump processes and Related Topics), Kyoto University, Departamental Bulletin Paper (2010), 1672: pp. 45-62, http://hdl.handle.net/2433/141183.

Tomasz Klimsiak and Andrzej Rozkosz Faculty of Mathematics and Computer Science Nicolaus Copernicus University

Chopina 12/18

87-100 Toruń

Poland

E-mail:tomas@mat.umk.pl

Andrzej Rozkosz

E-mail:rozkosz@mat.umk.pl 